\title{
ANALYSIS AND APPROXIMATION OF MIXED-DIMENSIONAL PDEs ON 3D-1D DOMAINS COUPLED WITH LAGRANGE MULTIPLIERS*
}

\author{
MIROSLAV KUCHTA ${ }^{\dagger}$, FEDERICA LAURINO $\ddagger$, KENT-ANDRE MARDAL ${ }^{\S}$, AND \\ PAOLO ZUNINO
}

\begin{abstract}
Coupled partial differential equations (PDEs) defined on domains with different dimensionality are usually called mixed-dimensional PDEs. We address mixed-dimensional PDEs on three-dimensional (3D) and one-dimensional (1D) domains, which gives rise to a 3D-1D coupled problem. Such a problem poses several challenges from the standpoint of existence of solutions and numerical approximation. For the coupling conditions across dimensions, we consider the combination of essential and natural conditions, which are basically the combination of Dirichlet and Neumann conditions. To ensure a meaningful formulation of such conditions, we use the Lagrange multiplier method suitably adapted to the mixed-dimensional case. The well-posedness of the resulting saddle-point problem is analyzed. Then, we address the numerical approximation of the problem in the framework of the finite element method. The discretization of the Lagrange multiplier space is the main challenge. Several options are proposed, analyzed, and compared, with the purpose of determining a good balance between the mathematical properties of the discrete problem and flexibility of implementation of the numerical scheme. The results are supported by evidence based on numerical experiments.
\end{abstract}

Key words. mixed-dimensional PDEs, finite element approximation, essential coupling conditions, Lagrange multipliers

AMS subject classifications. 35J25, 58J05, 65N30, 65N85

DOI. $10.1137 / 20 \mathrm{M} 1329664$

1. Introduction. In this study we consider coupled partial differential equations (PDEs) on domains with mixed dimensionality; in particular, we address mixeddimensional PDEs on three-dimensional (3D) and one-dimensional (1D) domains (3D$1 \mathrm{D}$ case). The mathematical structure of such problems can be represented by the following formal equations:

$$
\begin{aligned}
-\Delta u+u+\lambda \delta_{\Lambda} & =f & & \text { in } \Omega, \\
d_{s}^{2} u_{\odot}+u_{\odot}-\lambda & =g & & \text { on } \Lambda, \\
\mathcal{T}_{\Lambda} u-u_{\odot} & =q & & \text { on } \Lambda .
\end{aligned}
$$

Problem (1.1) can be described as an example of mixed-dimensional PDEs. Here, $u$, $u_{\odot}, \lambda$ are unknowns and $\Omega$ is a bounded domain in $\mathbb{R}^{3}$, whereas $\Lambda \subset \Omega$ is a $1 \mathrm{D}$ manifold parametrized in terms of $s$ and $d_{s}$ is the derivative with respect to $s$. The term $\lambda \delta_{\Lambda}$ is a Dirac measure such that $\int_{\Omega} \lambda(x) \delta_{\Lambda} v(x) \mathrm{d} x=\int_{\Lambda} \lambda(t) v(t) \mathrm{d} t$ for a continuous function $v$, and $\mathcal{T}_{\Lambda}: \Omega \rightarrow \Lambda$ is a suitable restriction operator from three dimensions to one dimension. We remark that $\lambda$ can be viewed as a Lagrange multiplier associated with

\footnotetext{
*Received by the editors April 6, 2020; accepted for publication (in revised form) December 7, 2020; published electronically February 23, 2021.

https://doi.org/10.1137/20M1329664

†Simula Research Laboratory, 1364 Fornebu, Norway (miroslav@simula.no).

${ }_{\ddagger}^{\ddagger}$ Department of Mathematics, MOX - Politecnico di Milano, Milano, 20133, Italy (federica. laurino@polimi.it).

$\S$ Department of Mathematics, University of Oslo, Oslo, 0316, Norway (kent-and@math.uio.no).

\Corresponding author. Department of Mathematics, MOX - Politecnico di Milano, Milano, 20133, Italy (paolo.zunino@polimi.it).
} 
the coupling constraint (1.1c); see section SM1 of the supplementary material for a precise definition.

Using models based on mixed-dimensional PDEs is motivated by the fact that many problems in geo- and biophysics are characterized by slender cylindrical structures coupled to a larger 3D body, where the characteristic transverse length scale of the slender structure is many orders of magnitude smaller than the longitudinal length. For example, in geophysical applications the radii of wells are often of the order of $10 \mathrm{~cm}$ while the length may be several $\mathrm{km}[27,28]$. Similarly, in applications involving blood flow and oxygen transport of microcirculation, the capillary radius is a few microns, while simulations are often performed on $\mathrm{mm}$ to $\mathrm{cm}$ scale, with thousands of vessels $[3,14,17,32]$. Finally, in neuroscience applications a neuron has a width of a few microns, while its length is much longer. For example, an axon of a motor neuron may be as long as a meter. Hence, at least four orders of magnitude in difference in the transverse and longitudinal directions is common in geophysics, biomechanics, and neuroscience. Meshes dictated by resolving the transverse length scale in three dimensions would then possibly lead to the order of $10^{12}$ degrees of freedom. Even if adaptive and strongly anisotropic meshes were allowed, the computations would quickly become demanding if many slender structures and their interactions were considered.

From a mathematical standpoint, the challenge involved in problem (1.1) is that neither $\mathcal{T}_{\Lambda}$ nor $\delta_{\Lambda}$ is well defined. That is, without extra regularity, solutions of elliptic PDEs only have well defined traces of codimension one. Here, $\mathcal{T}_{\Lambda}$ is of codimension two, mapping functions defined on a domain in three dimensions to functions defined along a $1 \mathrm{D}$ curve. The challenge of coupling PDEs on domains with high dimensionality gap has recently attracted the attention of many researchers. The works by D'Angelo [10, 11] and D'Angelo and Quarteroni [12] have remedied the well-posedness by weakening the solution concept. This approach naturally leads to nonsymmetric formulations. An alternative approach is to decompose the solution into smooth and nonsmooth components, where the nonsmooth component may be represented in terms of Green's functions, and then consider the well-posedness of the smooth component [16]. The numerical approximation of such equations has been also studied in a series of works. The consistent derivation of numerical approximation schemes for PDEs in mixed dimension is addressed in [5]. Concerning approximability, elliptic equations with Dirac sources represent an effective prototype that has been addressed in $[4,18,20]$, where the optimal a priori error estimates for the finite element approximation are derived. Furthermore, the interplay among the mathematical structure of the problem, its solvers, and preconditioners for its discretization was studied in detail in [22] for the solution of $1 \mathrm{D}$ differential equations embedded in two dimensions and more recently was extended to the 3D-1D case in [21].

Stemming from this literature, our work adopts and analyzes a different approach closely related to $[19,23]$. That is, we exploit the fact that $\Lambda$ is not strictly a $1 \mathrm{D}$ curve but rather a very thin $3 \mathrm{D}$ structure with a cross-sectional area far below what can be resolved. With this additional assumption, we show that robustness with respect to the cross-sectional area can be restored. The major novelty of this work is that we address essential coupling conditions, namely Dirichlet-Neumann conditions; see, in particular, problem (SM1.1) in the supplementary material. In previous works, for example, [12, 19, 23], natural coupling conditions of Robin-Robin type were analyzed. Dirichlet-type coupling conditions pose additional difficulties as the conditions are not a natural part of the weak formulation of the problem. As shown in section SM1 of the supplementary material, we overcome this difficulty by resorting to a weak formulation 
of the Dirichlet-Neumann coupling conditions across dimensions by using Lagrange multipliers.

Although the focus of the present work is mostly on the analysis and approximation of the proposed approach, we stress that it aims to build the mathematical foundations for tackling various applications involving 3D-1D mixed-dimensional PDEs, such as fluid-structure interaction of slender bodies [26], microcirculation and lymphatics [29, 33], subsurface flow models with wells [8], and the electrical activity of neurons.

2. Preliminaries. Let the domain $\Omega \subset \mathbb{R}^{3}$ be an open, connected, and convex set that can be subdivided into two parts $\Omega_{\ominus}$ and $\Omega_{\oplus}:=\Omega \backslash \bar{\Omega}_{\ominus}$. Let $\Omega_{\ominus}$ be a generalized cylinder (cf. [15]), that is, the swept volume of a two-dimensional set, $\partial \mathcal{D}$, moved along a curve, $\Lambda$, in the three-dimensional domain, $\Omega$; see Figure 2.1 for an illustration. More precisely, the curve $\Lambda=\{\boldsymbol{\lambda}(s), s \in(0, S)\}$, where $\boldsymbol{\lambda}(s)=[\xi(s), \tau(s), \zeta(s)]$, and $s \in(0, S)$ is a $\mathcal{C}^{2}$-regular curve in the three-dimensional domain $\Omega$. For simplicity, let us assume that $\left\|\boldsymbol{\lambda}^{\prime}(s)\right\|=1$ such that the arclength and the coordinate $s$ coincide. Further, let $\mathcal{D}(s)=[x(r, t), y(r, t)]:(0, R(s)) \times(0, T(s)) \rightarrow \mathbb{R}^{2}$ be a parametrization of the cross-section with $R(s) \geq R_{0}>0$ being $R_{0}$, that is, the minimum cross-sectional radius of the generalized cylinder, and let $\Gamma$ be the lateral surface of $\Omega_{\ominus}$, i.e., $\Gamma=\{\partial \mathcal{D}(s) \mid s \in \Lambda\}$, while the upper and lower faces of $\Omega_{\ominus}$ belong to $\partial \Omega$. We assume that $\Omega_{\ominus}$ crosses $\Omega$ from side to side. Finally, $|\cdot|$ denotes the Lebesgue measure of a set, e.g., $|\mathcal{D}(s)|$ is the cross-sectional area of the cylinder. In general, $|\mathcal{D}(s)|$ must be strictly positive and bounded. According to the geometrical setting, we will denote by $v, v_{\oplus}, v_{\ominus}, v_{\odot}$ functions defined on $\Omega, \Omega_{\oplus}, \Omega_{\ominus}, \Lambda$, respectively.

Let $D$ be a generic regular bounded domain in $\mathbb{R}^{3}$, and let $X$ be a Hilbert space defined on $D$. Then $(\cdot, \cdot)_{X}$ and $\|\cdot\|_{X}$ denote the inner product and norm of $X$, respectively. The duality pairing between the $X$ and its dual $X^{*}$ is denoted by $\langle\cdot, \cdot\rangle$. Let $(\cdot, \cdot)_{L^{2}(D)},(\cdot, \cdot)_{D}$, or simply $(\cdot, \cdot)$ be the $L^{2}(D)$ inner product on $D$. We use the standard notation $H^{q}(D)$ to denote the Sobolev space of functions on $D$ with all derivatives up to the order $q$ in $L^{2}(D)$. The corresponding norm is $\|\cdot\|_{H^{q}(D)}$, and the seminorm is $|\cdot|_{H^{q}(D)}$. The space $H_{0}^{q}(D)$ represents the closure in $H^{q}(D)$ of smooth functions with compact support in $D$.

Let $\Sigma$ be a Lipschitz codimension one subset of $D$. We denote by $\mathcal{T}_{\Sigma}: H^{q}(D) \rightarrow$ $H^{q-\frac{1}{2}}(\Sigma)$ the trace operator from $D$ to $\Sigma$. The space of functions in $H^{\frac{1}{2}}(\Sigma)$ with continuous extension by zero outside $\Sigma$ is denoted by $H_{00}^{\frac{1}{2}}(\Sigma)$, and we remark that $H_{00}^{\frac{1}{2}}(\Sigma)=\mathcal{T}_{\Sigma} H_{0}^{1}(D)$ and $H^{-\frac{1}{2}}(\Sigma)=\left(H_{00}^{\frac{1}{2}}(\Sigma)\right)^{*}$.

We will frequently use inner products and norms that are weighted. The $L_{2}$ and $H^{1}$ inner products weighted by a scalar function $w$, which is strictly positive and bounded almost everywhere, are defined as

$$
(u, v)_{L^{2}(\Sigma), w}=\int_{\Sigma} w u v d \omega \text { and }(u, v)_{H^{1}(\Sigma), w}=\int_{\Sigma} w u v d \omega+\int_{\Sigma} w \nabla u \cdot \nabla v d \omega
$$

whereas a weighted fractional space $H_{00}^{s}(\Sigma ; w)$ is defined in terms of the interpolation of the corresponding weighted spaces (see [24, Chap. 2.1] and also [2, 9]). More precisely, we have $H_{00}^{s}(\Gamma ; w)=\left[H_{0}^{1}(\Sigma ; w), L^{2}(\Sigma ; w)\right]_{s}$, with $s \in[0,1]$ from the notation of [2]. For the norm of such spaces, we introduce the Riesz map $S$ such that for $u, v \in H_{0}^{1}(\Sigma)$ we have

$$
\int_{\Sigma} w \nabla(S u) \cdot \nabla v d \omega=(u, v)_{L^{2}(\Sigma), w}
$$

Copyright (c) by SIAM. Unauthorized reproduction of this article is prohibited. 


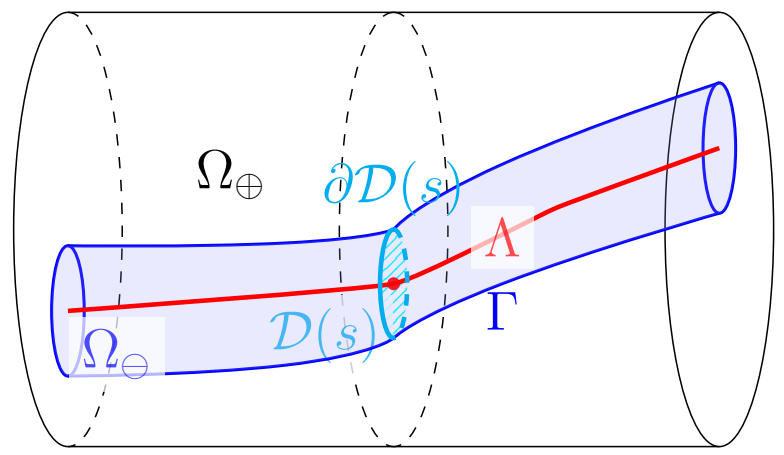

FIG. 2.1. Geometrical setting of the problem.

Then $S=-\Delta^{-1}$ is a compact self-adjoint operator. Assuming that $\left\{\lambda_{k}\right\}_{k}$ is the set of eigenvalues, $\left\{\phi_{k}\right\}_{k}$ is the set of eigenvectors of $S$ orthonormal with respect to the inner product $(\cdot, \cdot)_{L^{2}(\Sigma), w}$, and $u \in H_{0}^{1}(\Sigma)$ can be expressed as $u=\sum_{k} c_{k} \phi_{k}$, we have

$$
\|u\|_{H_{00}^{s}(\Sigma), w}^{2}=\sum_{k} \lambda_{k}^{-s} c_{k}^{2} .
$$

Owing to the positivity and boundedness of $w$, the weighted spaces are equal to the corresponding nonweighted spaces as sets, but their norms are different.

Central to our analysis are the transverse averages $\bar{w}, \overline{\bar{w}}$ defined as

$$
\bar{w}(s)=|\partial \mathcal{D}(s)|^{-1} \int_{\partial \mathcal{D}(s)} w d \gamma \quad \text { and } \quad \overline{\bar{w}}(s)=|\mathcal{D}(s)|^{-1} \int_{\mathcal{D}(s)} w d \sigma,
$$

where $d \omega, d \sigma, d \gamma$ are the generic volume, surface, and curvilinear Lebesgue measures. Clearly,

$$
\begin{gathered}
\int_{\Omega_{\ominus}} w d \omega=\int_{\Lambda} \int_{\mathcal{D}(s)} w d \sigma d s=\int_{\Lambda}|\mathcal{D}(s)| \overline{\bar{w}}(s) d s \\
\int_{\partial \Omega_{\ominus}} w d \sigma=\int_{\Lambda} \int_{\partial \mathcal{D}(s)} w d \gamma d s=\int_{\Lambda}|\partial \mathcal{D}(s)| \bar{w}(s) d s .
\end{gathered}
$$

Analogously, for functions defined on $\Lambda$ and $\Omega_{\ominus}$, respectively, we let $d_{s}$ and $\partial_{s}$ be the ordinary and partial derivatives with respect to the arclength.

The operator obtained from a combination of the average operator $\overline{(\cdot)}$ with the trace on $\Gamma$ will be denoted by $\overline{\mathcal{T}}_{\Lambda}=\overline{(\cdot)} \circ \mathcal{T}_{\Gamma}$, as it maps functions on $\Omega$ to functions on $\Lambda$. Further, let the extension operator $\mathcal{E}_{\Gamma}: H_{00}^{\frac{1}{2}}(\Lambda) \rightarrow H_{00}^{\frac{1}{2}}(\Gamma)$ be defined such that $\left(\mathcal{E}_{\Gamma} v_{\odot}\right)(x)=v_{\odot}(s)$ for any $x \in \partial \mathcal{D}(s)$. Then, the following identity shows that the transversal uniform extension operator is the inverse of the transversal average:

$$
\left\langle\overline{\mathcal{T}}_{\Lambda} u, v_{\odot}\right\rangle_{\Lambda,|\partial \mathcal{D}|}=\int_{\Lambda}|\partial \mathcal{D}|\left(\frac{1}{|\partial \mathcal{D}|} \int_{\partial \mathcal{D}} \mathcal{T}_{\Gamma} u d \gamma\right) v_{\odot} d s=\left\langle\mathcal{T}_{\Gamma} u, \mathcal{E}_{\Gamma} v_{\odot}\right\rangle_{\Gamma}
$$

With the above notation we are now able to formulate the 3D-1D-1D problem. The problem reads as follows: given $f \in L^{2}(\Omega), g \in L^{2}\left(\Omega_{\ominus}\right), q \in H_{00}^{\frac{1}{2}}(\Gamma)$ find 
$u \in H_{0}^{1}(\Omega), u_{\odot} \in H_{0}^{1}(\Lambda), \lambda_{\odot} \in H^{-\frac{1}{2}}(\Lambda)$ such that

$$
\begin{aligned}
(u, v)_{H^{1}(\Omega)}+\left\langle\overline{\mathcal{T}}_{\Lambda} v, \lambda_{\odot}\right\rangle_{\Lambda,|\partial \mathcal{D}|} & =(f, v)_{L^{2}(\Omega)} & \forall v \in H_{0}^{1}(\Omega), \\
\left(u_{\odot}, v_{\odot}\right)_{H^{1}(\Lambda),|\mathcal{D}|}-\left\langle v_{\odot}, \lambda_{\odot}\right\rangle_{\Lambda,|\partial \mathcal{D}|} & =\left(\overline{\bar{g}}, v_{\odot}\right)_{L^{2}(\Lambda),|\mathcal{D}|} & \forall v_{\odot} \in H_{0}^{1}(\Lambda), \\
\left\langle\overline{\mathcal{T}}_{\Lambda} u-u_{\odot}, \mu_{\odot}\right\rangle_{\Lambda,|\partial \mathcal{D}|} & =\left\langle\bar{q}, \mu_{\odot}\right\rangle_{\Lambda,|\partial \mathcal{D}|} & \forall \mu_{\odot} \in H^{-\frac{1}{2}}(\Lambda) .
\end{aligned}
$$

In addition to the 3D-1D-1D problem we will also consider an intermediate problem where the $3 \mathrm{D}$ and $1 \mathrm{D}$ problems are coupled at an intermediate 2D surface encapsulating the 1D structure. This is referred to as the 3D-1D-2D problem and reads as follows: given $f \in L^{2}(\Omega), g \in L^{2}\left(\Omega_{\ominus}\right), q \in H_{00}^{\frac{1}{2}}(\Gamma)$ find $u \in H_{0}^{1}(\Omega), u_{\odot} \in H_{0}^{1}(\Lambda), \lambda \in$ $H^{-\frac{1}{2}}(\Gamma)$ such that

$$
\begin{aligned}
(u, v)_{H^{1}(\Omega)}+\left\langle\mathcal{T}_{\Gamma} v, \lambda\right\rangle_{\Gamma} & =(f, v)_{L^{2}(\Omega)} & & \forall v \in H_{0}^{1}(\Omega), \\
\left(u_{\odot}, v_{\odot}\right)_{H^{1}(\Lambda),|\mathcal{D}|}-\left\langle\mathcal{E}_{\Gamma} v_{\odot}, \lambda\right\rangle_{\Gamma} & =\left(\overline{\bar{g}}, v_{\odot}\right)_{L^{2}(\Lambda),|\mathcal{D}|} & & \forall v_{\odot} \in H^{1}(\Lambda), \\
\left\langle\mathcal{T}_{\Gamma} u-\mathcal{E}_{\Gamma} u_{\odot}, \mu\right\rangle_{\Gamma} & =\left\langle q, \mu_{\odot}\right\rangle_{\Gamma} & & \forall \mu \in H^{-\frac{1}{2}}(\Gamma) .
\end{aligned}
$$

We conclude this section with analysis of a fundamental property for the problem formulation that we will address, namely the characterization of the regularity of the operator $\overline{\mathcal{T}}_{\Lambda}$. More precisely, we aim to show that $\overline{\mathcal{T}}_{\Lambda}: H_{0}^{1}(\Omega) \rightarrow H_{00}^{\frac{1}{2}}(\Lambda)$. This is a consequence of the following lemma.

Lemma 2.1. Let $\Gamma$ be a tensor product domain, $\Gamma=(0, X) \times(0, Y)$. For any regular $u(x, y)$ in $\Gamma$, let $\bar{u}(x)=\frac{1}{Y} \int_{0}^{Y} u(x, y) d y$. Then, for any $u \in H_{00}^{\frac{1}{2}}(\Gamma), \bar{u}(x) \in$ $H_{00}^{\frac{1}{2}}((0, X))$. Moreover, if $u(x, y) \in H_{00}^{\frac{1}{2}}(\Gamma)$ is constant with respect to $y$, namely $u(x, y)=u(x)$, then

$$
\|u\|_{H_{00}^{\frac{1}{2}}(\Gamma)}=Y\|u\|_{H_{00}^{\frac{1}{2}}(0, X)} .
$$

The proof of Lemma 2.1 is based on the representation of fractional norms in terms of the spectrum of the Laplace operator and subsequent standard arguments in harmonic analysis. The full proof is reported in the supplementary material for the sake of clarity.

Under the geometric assumptions stated above for $\Omega, \Gamma, \Lambda$, Lemma 2.1 implies the following result.

Corollary 2.2 (of Lemma 2.1). If $u \in H_{00}^{\frac{1}{2}}(\Gamma)$, then $\bar{u} \in H_{00}^{\frac{1}{2}}(\Lambda)$, and there exists a constant $C_{\Gamma}$, bounded independently of $\mathcal{D}$ and $\partial \mathcal{D}$, such that

$$
\|\bar{u}\|_{H_{00}^{\frac{1}{2}}(\Lambda),|\partial \mathcal{D}|} \leq C_{\Gamma}\|u\|_{H_{00}^{\frac{1}{2}}(\Gamma)} .
$$

Proof. Since $\Gamma$ is the surface of a generalized cylinder, it can be parametrized as a tensor product domain using a local coordinate system such as the Frenet frame. Then, Lemma 2.1 can be applied. The inequality above follows from inequality (SM2.3) in section SM2 of the supplementary material.

Furthermore, from the above corollary, it is clear that $\overline{\mathcal{T}}_{\Lambda}: H_{0}^{1}(\Omega) \rightarrow H_{00}^{\frac{1}{2}}(\Lambda)$.

3. Saddle-point problem analysis. Let $a: X \times X \rightarrow \mathbb{R}$ and $b: X \times Q \rightarrow \mathbb{R}$ be bilinear forms. Let us consider a general saddle-point problem of the following form:

Copyright (c) by SIAM. Unauthorized reproduction of this article is prohibited. 
find $u \in X, \lambda \in Q$ such that

$$
\begin{aligned}
a(u, v)+b(v, \lambda) & =c(v) \quad \forall v \in X, \\
b(u, \mu) & =d(\mu) \quad \forall \mu \in Q .
\end{aligned}
$$

The Brezzi conditions [6] ensure that the problem (3.1) is well-posed. For our purpose, we use the following particular version of the Brezzi conditions.

Theorem 3.1. Let $a(\cdot, \cdot): X \times X \rightarrow \mathbb{R}$ and $b(\cdot, \cdot): X \times Q \rightarrow \mathbb{R}$ be bounded bilinear forms satisfying the properties

$$
\begin{array}{rlrl}
a(u, u) & \geq \alpha\|u\|_{X}^{2}, & u \in X, \\
a(u, v) & \leq C_{a}\|u\|_{X}\|v\|_{X}, & u, v & \in X, \\
b(u, \mu) & \leq C_{b}\|u\|_{X}\|\mu\|_{Q}, \\
\sup _{v \in X} \frac{b(v, \mu)}{\|v\|_{X}} & \geq \beta\|\mu\|_{Q}, & u \in X, \mu \in Q,
\end{array}
$$

with positive constants $\alpha, \beta, C_{a}, C_{b}$. Then, there exists a unique solution $u \in X$, $\lambda \in Q$ of problem (3.1), and the following a priori estimates hold:

$$
\begin{aligned}
\|u\|_{X} & \leq \frac{1}{\alpha}\|c\|_{X^{\prime}}+\frac{1}{\beta}\left(1+\frac{C_{a}}{\alpha}\right)\|d\|_{Q^{\prime}}, \\
\|\lambda\|_{Q} & \leq \frac{1}{\beta}\left(1+\frac{C_{a}}{\alpha}\right)\|c\|_{X^{\prime}}+\frac{C_{a}}{\beta^{2}}\left(1+\frac{C_{a}}{\alpha}\right)\|d\|_{Q^{\prime}} .
\end{aligned}
$$

Here, the coercivity condition (3.2) applies to $X$, which is a particular case of Brezzi's original conditions. We also note that the constant $C_{b}$ does not play a role in the a priori estimates, but it is relevant in the a priori analysis of the numerical approximation error of the finite element method.

3.1. Problem 3D-1D-2D. We aim to find $u \in H_{0}^{1}(\Omega), u_{\odot} \in H_{0}^{1}(\Lambda), \lambda \in$ $H^{-\frac{1}{2}}(\Gamma)$, solutions of $(3.1)$, where

$$
\begin{aligned}
a\left(\left[u, u_{\odot}\right],\left[v, v_{\odot}\right]\right) & =(u, v)_{H^{1}(\Omega)}+\left(u_{\odot}, v_{\odot}\right)_{H^{1}(\Lambda),|\mathcal{D}|}, \\
b\left(\left[v, v_{\odot}\right], \mu\right) & =\left\langle\mathcal{T}_{\Gamma} v-\mathcal{E}_{\Gamma} v_{\odot}, \mu\right\rangle_{\Gamma} \\
c\left(\left[v, v_{\odot}\right]\right) & =(f, v)_{L^{2}(\Omega)}+\left(\overline{\bar{g}}, v_{\odot}\right)_{L^{2}(\Lambda),|\mathcal{D}|} \\
d(\mu) & =\langle q, \mu\rangle_{\Gamma}
\end{aligned}
$$

We prove that the conditions of Theorem 3.1 are fulfilled when we choose $X=H_{0}^{1}(\Omega) \times$ $H_{0}^{1}(\Lambda), Q=H^{-\frac{1}{2}}(\Gamma)$, where $X$ is equipped with the norm $\left\|\left[u, u_{\odot}\right]\right\|\left\|^{2}=\right\| u \|_{H^{1}(\Omega)}^{2}+$ $\left\|u_{\odot}\right\|_{H^{1}(\Lambda),|\mathcal{D}|}^{2}$. For this purpose, we recall the trace inequality relative to the operator $\mathcal{T}_{\Gamma}$; namely, for any $v \in H^{1}(\Omega)$ there exists a constant $C_{T}$ depending on the diameter of $\Omega$ such that $\left\|\mathcal{T}_{\Gamma} v\right\|_{H_{00}^{\frac{1}{2}(\Gamma)}} \leq C_{T}\|v\|_{H^{1}(\Omega)}$. We also define a lifting operator from $H_{00}^{1 / 2}(\Gamma)$ to $H_{0}^{1}(\Omega)$. First, we define the harmonic extension $\mathcal{H}_{\Omega_{\oplus}}$ from $H_{00}^{1 / 2}(\Gamma)$ to $H_{0}^{1}\left(\Omega_{\oplus}\right)$ such that $\mathcal{H}_{\Omega_{\oplus}} \xi=v$ for any $\xi \in H_{00}^{1 / 2}(\Gamma)$ with $v \in H_{0}^{1}\left(\Omega_{\oplus}\right)$. Further, for this operator there exists $C_{\Omega_{\oplus}} \in \mathbb{R}^{+}$, depending only on the diameter of $\Omega_{\oplus}$, such that $\|v\|_{H^{1}\left(\Omega_{\oplus}\right)} \leq C_{\Omega_{\oplus}}\|\xi\|_{H_{00}^{1 / 2}(\Gamma)}$. Now, to define an extension from $H_{0}^{1}\left(\Omega_{\oplus}\right)$ to $H_{0}^{1}(\Omega)$ we use the results of [30], in particular Theorem 2.3 for the specific case of a domain with a long hole such as $\Omega_{\oplus}$, where it is established that there exists a lifting operator 
$\mathcal{E}_{\Omega}$ from $H_{0}^{1}\left(\Omega_{\oplus}\right)$ to $H_{0}^{1}(\Omega)$ such that $\mathcal{E}_{\Omega} \xi=v$ for any $\xi \in H_{0}^{1}\left(\Omega_{\oplus}\right)$ with $v \in H_{0}^{1}(\Omega)$, and there exists $C_{\Omega} \in \mathbb{R}^{+}$such that $\|v\|_{H^{1}\left(\Omega_{\oplus}\right)} \leq C_{\Omega}\|\xi\|_{H^{1}(\Omega)}$ where $C_{\Omega}$ is a positive constant independent of the (minimal) radius of $\Gamma$.

LEMma 3.2. The bilinear forms of the problem 3D-1D-2D satisfy conditions (3.2)(3.5) with constants $\alpha=1, \beta=\left(C_{\Omega_{\oplus}} C_{\Omega}\right)^{-1}, C_{a}=1, C_{b}=C_{T}+\left(\frac{\max |\partial \mathcal{D}|}{\min |\mathcal{D}|}\right)^{\frac{1}{2}}$.

Proof. We need to establish the four Brezzi conditions. The bilinear form $a(\cdot, \cdot)$ is clearly bounded and coercive with constants $\alpha=C_{a}=1$ since for any $u=u_{\odot}$, $v=v_{\odot}$, we have

$$
a\left(\left[u, u_{\odot}\right],\left[v, v_{\odot}\right]\right)=(u, v)_{H^{1}(\Omega)}+\left(u_{\odot}, v_{\odot}\right)_{H^{1}(\Lambda),|\mathcal{D}|}=\|u\|_{H^{1}(\Omega)}^{2}+\left\|u_{\odot}\right\|_{H^{1}(\Lambda),|\mathcal{D}|}^{2} .
$$

Furthermore, the bilinear form $b(\cdot, \cdot)$ is bounded because

$$
\begin{aligned}
b\left(\left[v, v_{\odot}\right], \mu\right) & =\left\langle\mathcal{T}_{\Gamma} v-\mathcal{E}_{\Gamma} v_{\odot}, \mu\right\rangle_{\Gamma} \leq\left\|\mathcal{T}_{\Gamma} v-\mathcal{E}_{\Gamma} v_{\odot}\right\|_{H_{00}^{\frac{1}{2}(\Gamma)}}\|\mu\|_{H^{-\frac{1}{2}(\Gamma)}} \\
& \leq\left(\left\|\mathcal{T}_{\Gamma} v\right\|_{H_{00}^{\frac{1}{2}(\Gamma)}}+\left\|\mathcal{E}_{\Gamma} v_{\odot}\right\|_{H_{00}^{\frac{1}{2}(\Gamma)}}\right)\|\mu\|_{H^{-\frac{1}{2}}(\Gamma)} \\
& \leq\left(C_{T}\|v\|_{H^{1}(\Omega)}+\left\|\mathcal{E}_{\Gamma} v_{\odot}\right\|_{H^{1}(\Gamma)}\right)\|\mu\|_{H^{-\frac{1}{2}(\Gamma)}} \\
& \leq\left(C_{T}\|v\|_{H^{1}(\Omega)}+\left(\frac{\max |\partial \mathcal{D}|}{\min |\mathcal{D}|}\right)^{\frac{1}{2}}\left\|v_{\odot}\right\|_{H^{1}(\Lambda),|\mathcal{D}|}\right)\|\mu\|_{H^{-\frac{1}{2}(\Gamma)}} \\
& \leq\left(C_{T}+\left(\frac{\max |\partial \mathcal{D}|}{\min |\mathcal{D}|}\right)^{\frac{1}{2}}\right)\left\|\left[v, v_{\odot}\right]\right\|\|\mu\|_{H^{-\frac{1}{2}(\Gamma)}} .
\end{aligned}
$$

To fulfill the inf-sup condition for $b(\cdot, \cdot)$ we choose $v_{\odot} \in H_{0}^{1}(\Lambda)$ such that $\mathcal{E}_{\Gamma} v_{\odot}=0$. Therefore we obtain

$$
\sup _{\substack{v \in H_{0}^{1}(\Omega), v_{\odot} \in H_{0}^{1}(\Lambda)}} \frac{\left\langle\mathcal{T}_{\Gamma} v-\mathcal{E}_{\Gamma} v_{\odot}, \mu\right\rangle_{\Gamma}}{\left\|\left[v, v_{\odot}\right]\right\|} \geq \sup _{v \in H_{0}^{1}(\Omega)} \frac{\left\langle\mathcal{T}_{\Gamma} v, \mu\right\rangle_{\Gamma}}{\|v\|_{H^{1}(\Omega)}} .
$$

We note that the trace operator is surjective from $H_{0}^{1}(\Omega)$ to $H_{00}^{\frac{1}{2}}(\Gamma)$. Indeed, $\forall \xi \in$ $H_{00}^{\frac{1}{2}}(\Gamma)$, we can find $v=\mathcal{E}_{\Omega} \mathcal{H}_{\Omega_{\oplus}} \xi$. Using the stability of $\mathcal{E}_{\Omega}, \mathcal{H}_{\Omega_{\oplus}}$, we obtain

$$
\sup _{v \in H_{0}^{1}(\Omega)} \frac{\left\langle\mathcal{T}_{\Gamma} v, \mu\right\rangle_{\Gamma}}{\|v\|_{H^{1}(\Omega)}} \geq \sup _{\xi \in H_{00}^{\frac{1}{2}}(\Gamma)} \frac{\langle\xi, \mu\rangle_{\Gamma}}{C_{\Omega_{\oplus}} C_{\Omega}\|\xi\|_{H_{00}(\Gamma)}}=\left(C_{\Omega_{\oplus}} C_{\Omega}\right)^{-1}\|\mu\|_{H^{-\frac{1}{2}}(\Gamma)},
$$

where in the last inequality we exploited the fact that $H^{-\frac{1}{2}}(\Gamma)=\left(H_{00}^{\frac{1}{2}}(\Gamma)\right)^{*}$. Then, (3.5) is satisfied with $\beta=\left(C_{\Omega_{\oplus}} C_{\Omega}\right)^{-1}$, a constant independent of the size of the inclusion.

Corollary 3.3 (of Theorem 3.1). The 3D-1D-2D problem admits a unique solution $u \in H_{0}^{1}(\Omega), u_{\odot} \in H_{0}^{1}(\Lambda), \lambda \in H^{-\frac{1}{2}}(\Gamma)$ that satisfies the following a priori estimates, with constants independent of the minimal (transverse) diameter of $\Gamma$ :

$$
\begin{aligned}
\left\|\left[u, u_{\odot}\right]\right\| & \leq\left(\|f\|_{L^{2}(\Omega)}+\|\overline{\bar{g}}\|_{L^{2}(\Lambda),|\mathcal{D}|}\right)+2 C_{\Omega_{\oplus}} C_{\Omega}\|q\|_{H_{00}^{\frac{1}{2}(\Gamma)}}, \\
\|\lambda\|_{H^{-\frac{1}{2}}(\Gamma)} & \leq 2 C_{\Omega_{\oplus}} C_{\Omega}\left(\|f\|_{L^{2}(\Omega)}+\|\overline{\bar{g}}\|_{L^{2}(\Lambda),|\mathcal{D}|}\right)+2\left(C_{\Omega_{\oplus}} C_{\Omega}\right)^{2}\|q\|_{H_{00}^{\frac{1}{2}(\Gamma)}} .
\end{aligned}
$$

Copyright (c) by SIAM. Unauthorized reproduction of this article is prohibited. 
3.2. Problem 3D-1D-1D. We aim to find a solution $u \in H_{0}^{1}(\Omega), u_{\odot} \in H_{0}^{1}(\Lambda)$, $\lambda_{\odot} \in H^{-\frac{1}{2}}(\Lambda)$ of $(3.1)$ with

$$
\begin{aligned}
a\left(\left[u, u_{\odot}\right],\left[v, v_{\odot}\right]\right) & =(u, v)_{H^{1}(\Omega)}+\left(u_{\odot}, v_{\odot}\right)_{H^{1}(\Lambda),|\mathcal{D}|}, \\
b\left(\left[v, v_{\odot}\right], \mu_{\odot}\right) & =\left\langle\overline{\mathcal{T}}_{\Lambda} v-v_{\odot}, \mu_{\odot}\right\rangle_{\Lambda,|\partial \mathcal{D}|}, \\
c\left(\left[v, v_{\odot}\right]\right) & =(f, v)_{L^{2}(\Omega)}+\left(\overline{\bar{g}}, v_{\odot}\right)_{L^{2}(\Lambda),|\mathcal{D}|} \\
d\left(\mu_{\odot}\right) & =\left\langle\bar{q}, \mu_{\odot}\right\rangle_{\Lambda,|\partial \mathcal{D}|} .
\end{aligned}
$$

We prove that the assumptions of Theorem 3.1 are fulfilled with the spaces $X=$ $H_{0}^{1}(\Omega) \times H_{0}^{1}(\Lambda), Q=H^{-\frac{1}{2}}(\Lambda)$. Let us consider $X$ equipped with the norm $\|[\cdot, \cdot]\| \|$ and $Q$ equipped with the norm $\|\cdot\|_{H^{-\frac{1}{2}}(\Lambda),|\partial \mathcal{D}|}$.

LEMMA 3.4. The bilinear forms of the problem 3D-1D-1D satisfy conditions (3.2)(3.5) with constants $\alpha=1, \beta=\left(C_{\Omega_{\oplus}} C_{\Omega}\right)^{-1}, C_{a}=1, C_{b}=C_{\Gamma} C_{T}+\left(\frac{\max |\partial \mathcal{D}|}{\min |\mathcal{D}|}\right)^{\frac{1}{2}}$, where $C_{\Gamma}$ is the constant of Corollary 2.2.

Proof. The proof for the bilinear form $a(\cdot, \cdot)$ does not change with respect to the previous case.

The bound on $b(\cdot, \cdot)$ is established as

$$
\begin{gathered}
b\left(\left[v, v_{\odot}\right], \mu_{\odot}\right)=\left\langle\overline{\mathcal{T}}_{\Lambda} v-v_{\odot}, \mu_{\odot}\right\rangle_{\Lambda,|\partial \mathcal{D}|} \leq\left\|\overline{\mathcal{T}}_{\Lambda} v-v_{\odot}\right\|_{H_{00}^{\frac{1}{2}}(\Lambda),|\partial \mathcal{D}|}\left\|\mu_{\odot}\right\|_{H^{-\frac{1}{2}}(\Lambda),|\partial \mathcal{D}|} \\
\leq\left(\left\|\overline{\mathcal{T}}_{\Lambda} v\right\|_{H_{00}^{\frac{1}{2}}(\Lambda),|\partial \mathcal{D}|}+\left\|v_{\odot}\right\|_{H_{00}^{\frac{1}{2}}(\Lambda),|\partial \mathcal{D}|}\right)\left\|\mu_{\odot}\right\|_{H^{-\frac{1}{2}}(\Lambda),|\partial \mathcal{D}|} \\
\leq\left(C_{\Gamma}\left\|\mathcal{T}_{\Gamma} v\right\|_{H_{00}^{\frac{1}{2}}(\Gamma)}+\left\|v_{\odot}\right\|_{H^{1}(\Lambda),|\partial \mathcal{D}|}\right)\left\|\mu_{\odot}\right\|_{H^{-\frac{1}{2}}(\Lambda),|\partial \mathcal{D}|} \\
\leq\left(C_{\Gamma} C_{T}\|v\|_{H^{1}(\Omega)}+\left(\frac{\max |\partial \mathcal{D}|}{\min |\mathcal{D}|}\right)^{\frac{1}{2}}\left\|v_{\odot}\right\|_{H^{1}(\Lambda),|\mathcal{D}|}\right)\left\|\mu_{\odot}\right\|_{H^{-\frac{1}{2}}(\Lambda),|\partial \mathcal{D}|} \\
\leq\left(C_{\Gamma} C_{T}+\left(\frac{\max |\partial \mathcal{D}|}{\min |\mathcal{D}|}\right)^{\frac{1}{2}}\right)\left\|\left[v, v_{\odot}\right]\right\|\left\|\mu_{\odot}\right\|_{H^{-\frac{1}{2}}(\Lambda),|\partial \mathcal{D}|} .
\end{gathered}
$$

To show that the inf-sup condition holds, we choose $v_{\odot}=0$ and obtain

$$
\sup _{\substack{v \in H_{0}^{1}(\Omega), v \odot \in H_{0}^{1}(\Lambda)}} \frac{\left\langle\overline{\mathcal{T}}_{\Lambda} v-v_{\odot}, \mu_{\odot}\right\rangle_{\Lambda,|\partial \mathcal{D}|}}{\left\|\left[v, v_{\odot}\right]\right\| \mid} \sup _{v \in H_{0}^{1}(\Omega)} \frac{\left\langle\overline{\mathcal{T}}_{\Lambda} v, \mu_{\odot}\right\rangle_{\Lambda,|\partial \mathcal{D}|}}{\|v\|_{H^{1}(\Omega)}} .
$$

For any $q \in H_{00}^{\frac{1}{2}}(\Lambda)$, we consider the uniform extension to $\Gamma$ named as $\mathcal{E}_{\Gamma} q$, and then we consider the extension operator from $H_{00}^{\frac{1}{2}}(\Gamma)$ to $H_{0}^{1}(\Omega)$ defined before, namely $\mathcal{E}_{\Omega} \mathcal{H}_{\Omega_{\oplus}}$ such that $v=\mathcal{E}_{\Omega} \mathcal{H}_{\Omega_{\oplus}} \mathcal{E}_{\Gamma} q \in H_{0}^{1}(\Omega)$. It follows that for any $q \in H_{00}^{\frac{1}{2}}(\Lambda)$ there exists $v \in H_{0}^{1}(\Omega)$ such that $\overline{\mathcal{T}}_{\Lambda} v=q$. Therefore we have

$$
\sup _{v \in H_{0}^{1}(\Omega)}\left\langle\overline{\mathcal{T}}_{\Lambda} v, \mu_{\odot}\right\rangle_{\Lambda,|\partial \mathcal{D}|} \geq \sup _{q \in H_{00}^{\frac{1}{2}}(\Lambda)}\left\langle q, \mu_{\odot}\right\rangle_{\Lambda,|\partial \mathcal{D}|} .
$$

Moreover, using Lemma 2.1 we obtain

$$
\|v\|_{H_{0}^{1}(\Omega)} \leq C_{\Omega_{\oplus}} C_{\Omega}\left\|\mathcal{E}_{\Gamma} q\right\|_{H_{00}^{\frac{1}{2}(\Gamma)}}=C_{\Omega_{\oplus}} C_{\Omega}\|q\|_{H_{00}^{\frac{1}{2}}(\Lambda),|\partial \mathcal{D}|} .
$$

Copyright $@$ by SIAM. Unauthorized reproduction of this article is prohibited. 
We conclude the proof with the following inequalities:

$$
\begin{aligned}
& \sup _{v \in H_{0}^{1}(\Omega)} \frac{\left\langle\overline{\mathcal{T}}_{\Lambda} v, \mu_{\odot}\right\rangle_{\Lambda,|\partial \mathcal{D}|}}{\|v\|_{H^{1}(\Omega)}} \geq \sup _{q \in H_{00}^{\frac{1}{2}}(\Lambda)} \frac{\left\langle q, \mu_{\odot}\right\rangle_{\Lambda,|\partial \mathcal{D}|}}{\|v\|_{H^{1}(\Omega)}} \\
& \geq \frac{1}{C_{\Omega_{\oplus}} C_{\Omega}} \sup _{q \in H_{00}^{\frac{1}{2}}(\Lambda)} \frac{\left\langle q, \mu_{\odot}\right\rangle_{\Lambda,|\partial \mathcal{D}|}}{\|q\|_{H_{00}}^{\frac{1}{2}}(\Lambda),|\partial \mathcal{D}|}=\frac{1}{C_{\Omega_{\oplus}} C_{\Omega}}\left\|\mu_{\odot}\right\|_{H^{-\frac{1}{2}}(\Lambda),|\partial \mathcal{D}|} .
\end{aligned}
$$

Corollary 3.5 (of Theorem 3.1). The 3D-1D-1D problem admits a unique solution $u \in H_{0}^{1}(\Omega), u_{\odot} \in H_{0}^{1}(\Lambda), \lambda \in H^{-\frac{1}{2}}(\Lambda)$ that satisfies the following a priori estimates, with constants independent of the minimal (transverse) diameter of $\Gamma$ :

$$
\begin{aligned}
\left\|\left[u, u_{\odot}\right]\right\| \| & \leq\left(\|f\|_{L^{2}(\Omega)}+\|\overline{\bar{g}}\|_{L^{2}(\Lambda),|\mathcal{D}|}\right)+2 C_{\Omega_{\oplus}} C_{\Omega}\|\bar{q}\|_{H_{00}^{\frac{1}{2}}(\Lambda),|\partial \mathcal{D}|}, \\
\|\lambda\|_{H^{-\frac{1}{2}}(\Lambda),|\partial \mathcal{D}|} & \leq 2 C_{\Omega_{\oplus}} C_{\Omega}\left(\|f\|_{L^{2}(\Omega)}+\|\overline{\bar{g}}\|_{L^{2}(\Lambda),|\mathcal{D}|}\right)+2\left(C_{\Omega_{\oplus}} C_{\Omega}\right)^{2}\|\bar{q}\|_{H_{00}^{\frac{1}{2}}(\Lambda),|\partial \mathcal{D}|} .
\end{aligned}
$$

Remark 3.1. Corollaries 3.3 and 3.5 show that the stability of the continuous problem is not affected by the size of the inclusion, because all the stability constants are uniformly independent of $|\mathcal{D}|,|\partial \mathcal{D}|$. Referring, for example, to the 3D-1D-1D problem, formally taking the limit for $|\mathcal{D}|,|\partial \mathcal{D}| \rightarrow 0$, we observe that the weak formulation of the problem would tend to the trivial case $(u, v)_{H^{1}(\Omega)}=(f, v)_{L^{2}(\Omega)}$, and in a similar way the a priori estimates would consistently reduce to $\|u\|_{H^{1}(\Omega)} \leq\|f\|_{L^{2}(\Omega)}$. In other words, the weak formulation of the problem and the a priori estimates are robust for an arbitrary small size of the inclusion.

4. Finite element approximation. In this section we consider the discretization of the problems 3D-1D-2D and 3D-1D-1D by means of the finite element method. We address two main objectives: first, we aim to identify a suitable approximation space for the Lagrange multiplier and to analyze the stability of the discrete saddlepoint problem; second, we aim to derive a stable discretization method that uses independent computational meshes for $\Omega$ and $\Lambda$ not necessarily conforming to $\Gamma$. The latter objective is particularly relevant for the application of this approach in the case of very small inclusions, because it possibly allows us to use a computational mesh on $\Omega$ with a characteristic size $h$ that is larger than the (cross-sectional) diameter of the inclusion.

Let us introduce a shape-regular triangulation $\mathcal{T}_{h}^{\Omega}$ of $\Omega$ and an admissible partition $\mathcal{T}_{\mathfrak{h}}^{\Lambda}$ of $\Lambda$. We analyze two different cases: the conforming case, where compatibility constraints are satisfied by $\mathcal{T}_{h}^{\Omega}$ and $\mathcal{T}_{h}^{\Lambda}$ with respect to $\Gamma$ and consequently $h=\mathfrak{h}$; and the nonconforming case, where it is possible to choose $\mathcal{T}_{h}^{\Omega}$ and $\mathcal{T}_{\mathfrak{h}}^{\Lambda}$ arbitrarily.

Remark 4.1. The mesh conformity assumptions between $\mathcal{T}_{h}^{\Omega}, \mathcal{T}_{\mathfrak{h}}^{\Lambda}$, and $\Gamma$ (see below for a precise definition) necessarily imply that $h=\mathfrak{h} \leq R_{0}$, where $R_{0}$ is the minimum cross-sectional radius of the inclusion $\Omega_{\ominus}$ that is shaped as a generalized cylinder as shown in Figure 2.1.

4.1. Analysis of the case where $\mathcal{T}_{h}^{\Omega}$ conforms to $\mathcal{T}_{\mathfrak{h}}^{\Lambda}$ and to $\Gamma$. As conformity conditions between $\mathcal{T}_{h}^{\Omega}, \mathcal{T}_{\mathfrak{h}}^{\Lambda}$, and $\Gamma$, it is required that the intersection of $\mathcal{T}_{h}^{\Omega}$ and $\Gamma$ is made of entire faces of elements $K \in \mathcal{T}_{h}^{\Omega}$. Furthermore, we also set a restriction between $\mathcal{T}_{h}^{\Omega}$ and $\mathcal{T}_{\mathfrak{h}}^{\Lambda}$. We assume that $\Lambda$ is a piecewise linear manifold. For any internal node of $\mathcal{T}_{\mathfrak{h}}^{\Lambda}$, we want a cross-sectional plane intersecting $\Gamma$ to be defined. 
We require that all the nodes of $\mathcal{T}_{h}^{\Omega}$ lying on $\Gamma$ fall on the intersection of $\Gamma$ with such cross-sectional planes. As a result of the latter condition we have $h \simeq \mathfrak{h}$. For this reason, from now on throughout this section we denote $\mathcal{T}_{h}^{\Lambda}$ as the mesh on $\Lambda$.

In this case, the discrete equivalent of (3.1) reads as follows: find $u_{h} \in X_{h} \subset X$, $\lambda_{h} \in Q_{h} \subset Q$ such that

$$
\begin{array}{rlrl}
a\left(u_{h}, v_{h}\right)+b\left(v_{h}, \lambda_{h}\right) & =c\left(v_{h}\right) & & \forall v_{h} \in X_{h}, \\
b\left(u_{h}, \mu_{h}\right) & =d\left(\mu_{h}\right) & \forall \mu_{h} \in Q_{h},
\end{array}
$$

where, with slight abuse of notation, we use $h$ as the subindex for all the discretization spaces. This discrete problem is well-posed if the conditions (3.2)-(3.5) apply to $X_{h}$ and $Q_{h}$. Since $X_{h} \subset X$ and $Q_{h} \subset Q,(3.2)-(3.4)$ follow immediately, and only the infsup condition needs consideration; see, for example, [13, Theorem 2.42]. Furthermore, Ceá type approximation estimates can be easily derived, as shown in [13, Theorem 2.44]. We summarize these results in the theorem below.

Theorem 4.1. Let $X_{h} \subset X, Q_{h} \subset Q, a(\cdot, \cdot)$, and $b(\cdot, \cdot)$ satisfy the conditions (3.2)-(3.4); then the problem (4.1) is well-posed if the discrete counterpart of (3.5) is satisfied, i.e., there exists a constant $\beta_{h}>0$, independent of the mesh discretization size $h$, such that

$$
\sup _{v_{h} \in X_{h}} \frac{b\left(v_{h}, \mu_{h}\right)}{\left\|v_{h}\right\|_{X}} \geq \beta_{h}\left\|\mu_{h}\right\|_{Q} \quad \forall \mu_{h} \in Q_{h} .
$$

Furthermore the following a priori error estimates hold:

$$
\begin{aligned}
\left\|u-u_{h}\right\|_{X} & \leq\left(1+\frac{C_{a}}{\alpha}\right)\left(1+\frac{C_{b}}{\beta_{h}}\right) \inf _{v_{h} \in X_{h}}\left\|u-v_{h}\right\|_{X}+\frac{C_{b}}{\alpha} \inf _{\mu_{h} \in Q_{h}}\left\|\lambda-\mu_{h}\right\|_{Q}, \\
\left\|\lambda-\lambda_{h}\right\|_{Q} & \leq \frac{C_{a}}{\beta_{h}}\left(1+\frac{C_{a}}{\alpha}\right)\left(1+\frac{C_{b}}{\beta_{h}}\right) \inf _{v_{h} \in X_{h}}\left\|u-v_{h}\right\|_{X} \\
& +\left(1+\frac{C_{b}}{\beta_{h}}+\frac{C_{b}}{\alpha} \frac{C_{a}}{\beta_{h}}\right) \inf _{\mu_{h} \in Q_{h}}\left\|\lambda-\mu_{h}\right\|_{Q} .
\end{aligned}
$$

Before proceeding we state an auxiliary result used in the forthcoming analysis. From now on, $C$ denotes a generic constant independent of the mesh size.

LEMMA 4.2. Let $\mathcal{P}_{h}: H_{00}^{\frac{1}{2}}(\Sigma ; w) \rightarrow Q_{h}$ be the orthogonal projection operator defined for any $v \in H_{00}^{\frac{1}{2}}(\Sigma ; w)$ by $\left(\mathcal{P}_{h} v, \psi_{h}\right)_{\Sigma, w}=\left(v, \psi_{h}\right)_{\Sigma, w}$ for any $\psi_{h} \in Q_{h}$, where $w$ is a bounded and positive weight function. Then, $\mathcal{P}_{h}$ is continuous on $H_{00}^{\frac{1}{2}}(\Sigma ; w)$, namely $\left\|\mathcal{P}_{h} v\right\|_{H_{00}^{\frac{1}{2}}(\Sigma), w} \leq C\|v\|_{H_{00}^{\frac{1}{2}}(\Sigma), w}$.

Proof. We show that $\mathcal{P}_{h}$ is continuous on $L^{2}(\Sigma ; w)$ and on $H_{0}^{1}(\Sigma ; w)$ following [13, section 1.6.3]. Then, the desired result can be proved by interpolation between spaces, since $H_{00}^{\frac{1}{2}}(\Sigma ; w)=\left[H_{0}^{1}(\Sigma ; w), L^{2}(\Sigma ; w)\right]_{\frac{1}{2}}$, namely the interpolation space between $L^{2}(\Sigma ; w)$ and $H_{0}^{1}(\Sigma ; w)$. For the $L^{2}$-continuity, we exploit the fact that, from the definition of $\mathcal{P}_{h},\left(v-\mathcal{P}_{h} v, \mathcal{P}_{h} v\right)_{\Sigma, w}=0$. Therefore, by the Pythagorean identity,

$$
\|v\|_{L^{2}(\Sigma), w}^{2}=\left\|v-\mathcal{P}_{h} v\right\|_{L^{2}(\Sigma), w}^{2}+\left\|\mathcal{P}_{h} v\right\|_{L^{2}(\Sigma), w}^{2} \geq\left\|\mathcal{P}_{h} v\right\|_{L^{2}(\Sigma), w}^{2} .
$$

Let us now consider $v \in H_{0}^{1}(\Sigma ; w)$. The Scott-Zhang interpolation operator $\mathcal{S Z}_{h}$ from $H_{0}^{1}(\Sigma ; w)$ to $Q_{h}$ satisfies the following inequalities (see [31]; also see [13, Lemma 
1.130] and inequalities (i) and (ii) for the proof of (4.3) and (4.4), respectively):

$$
\begin{aligned}
\left\|\mathcal{S Z}_{h} v\right\|_{H^{1}(\Sigma), w} & \leq C_{1}\|v\|_{H^{1}(\Sigma), w}, \\
\left\|v-\mathcal{S Z}_{h} v\right\|_{L^{2}(\Sigma), w} & \leq C_{2} h\|v\|_{H^{1}(\Sigma), w} .
\end{aligned}
$$

Therefore, using (4.3), (4.4), the $L^{2}$-stability of $\mathcal{P}_{h}$, and the discrete inverse inequality, we obtain

$$
\begin{aligned}
\left\|\nabla \mathcal{P}_{h} v\right\|_{L^{2}(\Sigma), w} & \leq\left\|\nabla\left(\mathcal{P}_{h} v-\mathcal{S Z}_{h} v\right)\right\|_{L^{2}(\Sigma), w}+\left\|\nabla \mathcal{S Z}_{h} v\right\|_{L^{2}(\Sigma), w} \\
& \leq\left\|\nabla\left(\mathcal{P}_{h} v-\mathcal{S Z}_{h} v\right)\right\|_{L^{2}(\Sigma), w}+C_{1}\|v\|_{H^{1}(\Sigma), w} \\
& \leq \frac{C_{3}}{h}\left\|\mathcal{P}_{h}\left(v-\mathcal{S Z}_{h} v\right)\right\|_{L^{2}(\Sigma), w}+C_{1}\|v\|_{H^{1}(\Sigma), w} \\
& \leq \frac{C_{3}}{h}\left\|v-\mathcal{S} \mathcal{Z}_{h} v\right\|_{L^{2}(\Sigma), w}+C_{1}\|v\|_{H^{1}(\Sigma), w} \\
& \leq\left(C_{2} C_{3}+C_{1}\right)\|v\|_{H^{1}(\Sigma), w} .
\end{aligned}
$$

As a result of the previous inequalities we obtain that

$$
\left\|\mathcal{P}_{h} v\right\|_{L^{2}(\Sigma), w}^{2} \leq C\|v\|_{L^{2}(\Sigma), w}^{2}, \quad\left\|\mathcal{P}_{h} v\right\|_{H^{1}(\Sigma), w} \leq C\|v\|_{H^{1}(\Sigma), w}^{2} .
$$

It remains to show that $\left\|\mathcal{P}_{h} v\right\|_{H_{00}^{\frac{1}{2}}(\Sigma), w} \leq C\|v\|_{H_{00}^{\frac{1}{2}(\Sigma), w}}$. To this end we use the interpolation theory for operators in Banach spaces. Given two separable Hilbert spaces, let us denote by $\mathcal{L}(X, Y)$ the space of continuous linear operators from $X$ to $Y$. Then, by $L^{2}$ - and $H^{1}$-continuity of $\mathcal{P}_{h}$ we have that $\mathcal{P}_{h} \in \mathcal{L}\left(L^{2}(\Sigma ; w), L^{2}(\Sigma ; w)\right) \cap$ $\mathcal{L}\left(H_{0}^{1}(\Sigma ; w), H_{0}^{1}(\Sigma ; w)\right)$. Recalling that we define $H_{00}^{1 / 2}(\Sigma ; w)=\left[H_{0}^{1}(\Sigma ; w), L^{2}(\Sigma ; w)\right]_{\frac{1}{2}}$ and applying [2, Theorem 2.2] it follows that $P_{h} \in \mathcal{L}\left(H_{00}^{1 / 2}(\Sigma ; w), H_{00}^{1 / 2}(\Sigma ; w)\right)$, which implies the desired inequality. We remark that [2, Theorem 2.2] applies directly to our setting, as the interpolation spaces therein are considered with the spectral norm rather than the $K$-interpolation norm.

4.1.1. Problem 3D-1D-2D. We denote by $X_{h, 0}^{k}(\Omega) \subset H_{0}^{1}(\Omega)$, with $k>0$, the conforming finite element space of continuous piecewise polynomials of degree $k$ defined on $\Omega$ which satisfy homogeneous Dirichlet conditions on the boundary and denote by $X_{h, 0}^{k}(\Lambda) \subset H_{0}^{1}(\Lambda)$ the space of continuous piecewise polynomials of degree $k$ defined on $\Lambda$ which satisfy homogeneous Dirichlet conditions on $\Lambda \cap \partial \Omega$. The space $Q_{h}$ must be suitably chosen such that (4.2) holds. Let $Q_{h}$ be the trace space of $X_{h, 0}^{k}(\Omega)$, namely the space of continuous piecewise polynomials of degree $k$ defined on $\Gamma$ which satisfy homogeneous Dirichlet conditions on $\partial \Omega$. As a result, $Q_{h}=X_{h, 0}^{k}(\Gamma) \subset H_{00}^{\frac{1}{2}}(\Gamma)$. The discrete version of the 3D-1D-2D problem is as follows: find $u_{h} \in X_{h, 0}^{k}(\Omega), u_{\odot_{h}} \in$ $X_{h, 0}^{k}(\Lambda), \lambda_{h} \in Q_{h} \subset H^{-\frac{1}{2}}(\Gamma)$ such that

$$
\begin{aligned}
& \left(u_{h}, v_{h}\right)_{H^{1}(\Omega)}+\left(u_{\odot_{h}}, v_{\odot_{h}}\right)_{H^{1}(\Lambda),|\mathcal{D}|}+\left\langle\mathcal{T}_{\Gamma} v_{h}-\mathcal{E}_{\Lambda} v_{\odot_{h}}, \lambda_{h}\right\rangle_{\Gamma} \\
& \quad=\left(f, v_{h}\right)_{L^{2}(\Omega)}+\left(\overline{\bar{g}}, v_{\odot_{h}}\right)_{L^{2}(\Lambda),|\mathcal{D}|} \quad \forall v_{h} \in X_{h, 0}^{k}(\Omega), v_{\odot_{h}} \in X_{h, 0}^{k}(\Lambda), \\
& \left\langle\mathcal{T}_{\Gamma} u_{h}-\mathcal{E}_{\Lambda} u_{\odot_{h}}, \mu_{h}\right\rangle_{\Gamma}=\left\langle q, \mu_{h}\right\rangle_{\Gamma} \quad \forall \mu_{h} \in Q_{h} .
\end{aligned}
$$

In what follows, we analyze the well-posedness of the discrete problem.

Lemma 4.3. There exists a constant $\gamma_{h, 1}>0$ such that for any $\mu_{h} \in Q_{h}$

$$
\sup _{q_{h} \in Q_{h}} \frac{\left\langle q_{h}, \mu_{h}\right\rangle}{\left\|q_{h}\right\|_{H_{00}^{\frac{1}{2}}(\Gamma)}} \geq \gamma_{h, 1}\left\|\mu_{h}\right\|_{H^{-\frac{1}{2}}(\Gamma)} .
$$

Copyright $@$ by SIAM. Unauthorized reproduction of this article is prohibited. 
As for the inf-sup constants, we note that $\gamma_{h, 1}$ depends on the discrete functional spaces but is uniformly independent of the mesh characteristic size $h$.

Proof. From the continuous case, in particular from (3.8), we have

$$
\left(C_{\Omega_{\oplus}} C_{\Omega}\right)^{-1}\left\|\mu_{h}\right\|_{H^{-\frac{1}{2}}(\Gamma)} \leq \sup _{v \in H_{0}^{1}(\Omega)} \frac{\left\langle\mathcal{T}_{\Gamma} v, \mu_{h}\right\rangle}{\|v\|_{H^{1}(\Omega)}} \quad \forall \mu_{h} \in Q_{h},
$$

and by the trace inequality $\left\|\mathcal{T}_{\Gamma} v\right\|_{H^{\frac{1}{2}(\Gamma)}} \leq C_{T}\|v\|_{H^{1}(\Omega)}$ (see $[1$, section 7.56$]$ ), we obtain

$$
\sup _{v \in H_{0}^{1}(\Omega)} \frac{\left\langle\mathcal{T}_{\Gamma} v, \mu_{h}\right\rangle}{\|v\|_{H^{1}(\Omega)}} \leq C_{T} \sup _{v \in H_{0}^{1}(\Omega)} \frac{\left\langle\mathcal{T}_{\Gamma} v, \mu_{h}\right\rangle}{\left\|\mathcal{T}_{\Gamma} v\right\|_{H_{00}}^{\frac{1}{2}}(\Gamma)} .
$$

Using Lemma 4.2 with $\Sigma=\Gamma$ and $w=1$, we obtain

$$
\begin{aligned}
C_{T} \sup _{v \in H_{0}^{1}(\Omega)} \frac{\left\langle\mathcal{T}_{\Gamma} v, \mu_{h}\right\rangle}{\left\|\mathcal{T}_{\Gamma} v\right\|_{H_{00}^{\frac{1}{2}}(\Gamma)}}= & C_{T} \sup _{v \in H_{0}^{1}(\Omega)} \frac{\left\langle\mathcal{P}_{h}\left(\mathcal{T}_{\Gamma} v\right), \mu_{h}\right\rangle}{\left\|\mathcal{T}_{\Gamma} v\right\|_{H_{00}^{\frac{1}{2}}(\Gamma)}} \\
& \leq C \sup _{v \in H_{0}^{1}(\Omega)} \frac{\left\langle\mathcal{P}_{h}\left(\mathcal{T}_{\Gamma} v\right), \mu_{h}\right\rangle}{\left\|\mathcal{P}_{h}\left(\mathcal{T}_{\Gamma} v\right)\right\|_{H_{00}^{\frac{1}{2}}(\Gamma)}}=C \sup _{q_{h} \in Q_{h}} \frac{\left\langle q_{h}, \mu_{h}\right\rangle}{\left\|q_{h}\right\|_{H_{00}}{ }^{\frac{1}{2}}(\Gamma)}
\end{aligned}
$$

Theorem 4.4 (discrete inf-sup). The inequality (4.2) holds true; namely, there exists a positive constant $\beta_{h, 1}$ such that

$$
\sup _{\substack{v_{h} \in X_{h, 0}^{k}(\Omega), v_{\odot_{h}} \in X_{h, 0}^{k}(\Lambda)}} \frac{\left\langle\mathcal{T}_{\Gamma} v_{h}-\mathcal{E}_{\Gamma} v_{\odot h}, \mu_{h}\right\rangle_{\Gamma}}{\left.\|\left[v_{h}, v_{\odot}\right]\right]\|\| \mu_{h} \|_{H^{-\frac{1}{2}}(\Gamma)}} \geq \beta_{h, 1} \quad \forall \mu_{h} \in Q_{h} .
$$

Proof. As in the continuous case, we choose $v_{\odot h}=0$, and we have

$$
\sup _{\substack{v_{h} \in X_{h, 0}^{k}(\Omega), v_{\odot_{h}} \in X_{h, 0}^{k}(\Lambda)}} \frac{\left\langle\mathcal{T}_{\Gamma} v_{h}-\mathcal{E}_{\Gamma} v_{\odot}, \mu_{h}\right\rangle_{\Gamma}}{\left\|\left[v_{h}, v_{\odot}\right]\right\|} \geq \sup _{v_{h} \in X_{h, 0}^{k}(\Omega)} \frac{\left\langle\mathcal{T}_{\Gamma} v_{h}, \mu_{h}\right\rangle_{\Gamma}}{\left\|v_{h}\right\|_{H^{1}(\Omega)}} .
$$

Following the approach of the continuous case, we need to construct an extension operator from $Q_{h}$ to $X_{h, 0}^{k}(\Omega)$. Thanks to the conformity of $\mathcal{T}_{h}^{\Omega}$ to the interface $\Gamma$, the existence and stability of such an extension operator, named $\mathcal{E}_{\Omega}^{h}$ (as it is the discrete analogue of $\mathcal{E}_{\Omega} \mathcal{H}_{\Omega_{\oplus}}$ used before), is proved using the results of [34]. In particular, as $\Gamma$ splits $\Omega$ into $\Omega_{\oplus}$ and $\Omega_{\ominus}$ and as the corresponding meshes comply with this partition, we introduce $\mathcal{E}_{\Omega_{\oplus}}^{h}$ and $\mathcal{E}_{\Omega_{\ominus}}^{h}$ as the extension operators from $Q_{h}$ to $X_{h, 0}^{k}\left(\Omega_{\oplus}\right)$ and $X_{h}^{k}\left(\Omega_{\ominus}\right)$, respectively. Then, we set (with slight abuse of notation) $\mathcal{E}_{\Omega}^{h} q_{h}:=\left(\mathcal{E}_{\Omega_{\oplus}}^{h} q_{h}+\mathcal{E}_{\Omega_{\ominus}}^{h} q_{h}+\mathcal{T}_{\Gamma} q_{h}\right) \in X_{h, 0}^{k}(\Omega)$. By definition, we obtain that $\mathcal{T}_{\Gamma} \mathcal{E}_{\Omega}^{h}$ is the identity operator on $Q_{h}$ and, owing to the results of [34], there exists a constant $C_{\mathcal{D}}$ uniformly independent of $h$ but possibly dependent on the size of the inclusion, namely $\operatorname{diam}(\mathcal{D})$, such that $\left\|\mathcal{E}_{\Omega}^{h} q_{h}\right\|_{H^{1}(\Omega)} \leq C_{\mathcal{D}}\left\|q_{h}\right\|_{H_{00}^{\frac{1}{2}(\Gamma)}}$.

Using Lemma 4.3 and the boundedness of the extension operator $\mathcal{E}_{\Omega}^{h}$, we have

$$
\gamma_{h, 1}\left\|\mu_{h}\right\|_{H^{-\frac{1}{2}}(\Gamma)} \leq \sup _{q_{h} \in Q_{h}} \frac{\left\langle q_{h}, \mu_{h}\right\rangle_{\Gamma}}{\left\|q_{h}\right\|_{H_{00}^{\frac{1}{2}}(\Gamma)}} \leq C_{\mathcal{D}} \sup _{q_{h} \in Q_{h}} \frac{\left\langle q_{h}, \mu_{h}\right\rangle_{\Gamma}}{\left\|\mathcal{E}_{\Omega}^{h} q_{h}\right\|_{H^{1}(\Omega)}} .
$$

Copyright $\odot$ by SIAM. Unauthorized reproduction of this article is prohibited. 
Then, for any $q_{h} \in Q_{h}$ we have $q_{h}=\mathcal{T}_{\Gamma} \mathcal{E}_{\Omega}^{h} q_{h}$, and owing to this property we obtain the following inequality, which proves the condition, with $\beta_{h, 1}=\gamma_{h, 1} C_{\mathcal{D}}^{-1}$ :

$$
\begin{aligned}
\gamma_{h, 1}\left\|\mu_{h}\right\|_{H^{-\frac{1}{2}}(\Gamma)} \leq \sup _{q_{h} \in Q_{h}} \frac{\left\langle q_{h}, \mu_{h}\right\rangle_{\Gamma}}{\left\|q_{h}\right\|_{H_{00} \frac{1}{2}(\Gamma)}} \leq C_{\mathcal{D}} \sup _{q_{h} \in Q_{h}} \frac{\left\langle q_{h}, \mu_{h}\right\rangle_{\Gamma}}{\left\|\mathcal{E}_{\Omega}^{h} q_{h}\right\|_{H^{1}(\Gamma)}} \\
=C_{\mathcal{D}} \sup _{q_{h} \in Q_{h}} \frac{\left\langle\mathcal{T}_{\Gamma} \mathcal{E}_{\Omega}^{h} q_{h}, \mu_{h}\right\rangle_{\Gamma}}{\left\|\mathcal{E}_{\Omega}^{h} q_{h}\right\|_{H^{1}(\Omega)}} \leq C_{\mathcal{D}} \sup _{v_{h} \in X_{h, 0}^{k}(\Omega)} \frac{\left\langle\mathcal{T}_{\Gamma} v_{h}, \mu_{h}\right\rangle_{\Gamma}}{\left\|v_{h}\right\|_{H^{1}(\Omega)}}
\end{aligned}
$$

Corollary 4.5 (of Theorem 4.1). Problem (4.5) admits a unique solution $u_{h} \in$ $X_{h, 0}^{k}(\Omega), u_{\odot_{h}} \in X_{h, 0}^{k}(\Lambda), \lambda_{h} \in X_{h, 0}^{k}(\Gamma)$, and the a priori error estimates

$$
\begin{aligned}
\left\|\left[u-u_{h}, u_{\odot}-u_{\odot}\right]\right\| & \leq C_{1, \mathcal{D}} \mathcal{E} \mathcal{R} \mathcal{R}\left(u, u_{\odot}, \lambda\right), \\
\left\|\lambda-\lambda_{h}\right\|_{H^{-\frac{1}{2}}(\Gamma)} & \leq C_{2, \mathcal{D}} \mathcal{E} \mathcal{R} \mathcal{R}\left(u, u_{\odot}, \lambda\right)
\end{aligned}
$$

are satisfied, where $C_{1, \mathcal{D}}, C_{2, \mathcal{D}} \simeq\left(\frac{\max |\partial \mathcal{D}|}{\min |\mathcal{D}|}\right)^{\frac{1}{2}}$, and $\mathcal{E R} \mathcal{R}\left(u, u_{\odot}, \lambda\right)$ is the approximation error

$$
\mathcal{E R \mathcal { R }}\left(u, u_{\odot}, \lambda\right)=\inf _{\substack{v_{h} \in X_{h, 0}^{k}(\Omega) \\ v_{\odot_{h}} \in X_{h, 0}^{k}(\Lambda)}}\left\|\left[u-v_{h}, u_{\odot}-v_{\odot}\right]\right\|\left\|+\inf _{\mu_{h} \in X_{h, 0}^{k}(\Gamma)}\right\| \lambda-\mu_{h} \|_{H^{-\frac{1}{2}}(\Gamma)} .
$$

4.1.2. Problem 3D-1D-1D. In this case, we use the spaces $X_{h, 0}^{k}(\Omega), X_{h, 0}^{k}(\Lambda)$ defined previously. For the multiplier space we choose $Q_{h}=X_{h, 0}^{k}(\Lambda)$; therefore we impose homogeneous Dirichlet boundary conditions on $\Lambda \cap \partial \Omega$ also for the Lagrange multiplier. We aim to find $u_{h} \in X_{h, 0}^{k}(\Omega), u_{\odot h} \in X_{h, 0}^{k}(\Lambda), \lambda_{\odot h} \in Q_{h} \subset H^{-\frac{1}{2}}(\Lambda)$ such that

$$
\begin{aligned}
& \left(u_{h}, v_{h}\right)_{H^{1}(\Omega)}+\left(u_{\odot h}, v_{\odot h}\right)_{H^{1}(\Lambda),|\mathcal{D}|}+\left\langle\overline{\mathcal{T}}_{\Lambda} v_{h}-v_{\odot h}, \lambda_{\odot h}\right\rangle_{\Lambda,|\partial \mathcal{D}|} \\
& \quad=\left(f, v_{h}\right)_{L^{2}(\Omega)}+\left(\overline{\bar{g}}, v_{\odot h}\right)_{L^{2}(\Lambda),|\mathcal{D}|} \quad \forall v_{h} \in X_{h}(\Omega), v_{\odot h} \in X_{h}(\Lambda), \\
& \left\langle\overline{\mathcal{T}}_{\Lambda} u_{h}-u_{\odot h}, \mu_{\odot h}\right\rangle_{\Lambda,|\partial \mathcal{D}|}=\left\langle\bar{q}, \mu_{\odot h}\right\rangle_{\Lambda,|\partial \mathcal{D}|} \quad \forall \mu_{\odot h} \in Q_{h} .
\end{aligned}
$$

Below we address the well-posedness of the 3D-1D-1D discrete problem with this alternative choice of multiplier space.

Lemma 4.6. There exists a constant $\gamma_{h, 2}>0$ such that for any $\mu_{h} \in Q_{h}$,

$$
\sup _{q_{h} \in Q_{h}} \frac{\left\langle q_{h}, \mu_{\odot h}\right\rangle_{\Lambda,|\partial \mathcal{D}|}}{\left\|q_{h}\right\|_{H_{00}^{\frac{1}{2}}(\Lambda),|\partial \mathcal{D}|}} \geq \gamma_{h, 2}\left\|\mu_{\odot h}\right\|_{H^{-\frac{1}{2}}(\Lambda),|\partial \mathcal{D}|} .
$$

The proof of this lemma follows that of Lemma 4.2, used with $\Sigma=\Lambda$ and $w=$ $|\partial \mathcal{D}|$, and that of Lemma 4.3, with the only difference being that the arguments are applied to $\Lambda$ instead of $\Gamma$.

ThEOREM 4.7 (discrete inf-sup). Inequality (4.2) holds; namely, there exists a positive constant $\beta_{h, 2}$ such that

$$
\sup _{\substack{v_{h} \in X_{h, 0}^{k}(\Omega) \\ v_{\odot h} \in X_{h, 0}^{k}(\Lambda)}} \frac{\left\langle\overline{\mathcal{T}}_{\Lambda} v_{h}-v_{\odot h}, \mu_{\odot h}\right\rangle_{\Lambda,|\partial \mathcal{D}|}}{\left\|\left[v_{h}, v_{\odot h}\right]\right\|\|\| \mu_{\odot h} \|_{H^{-\frac{1}{2}}(\Lambda),|\partial \mathcal{D}|}} \geq \beta_{h, 2} \quad \forall \mu_{\odot h} \in Q_{h} .
$$

Copyright (c) by SIAM. Unauthorized reproduction of this article is prohibited. 
Proof. Again, we choose $v_{\odot h}=0$, so that the proof reduces to showing that there exists $\beta_{h, 2}$ such that

$$
\sup _{v_{h} \in X_{h, 0}^{k}(\Omega)} \frac{\left\langle\overline{\mathcal{T}}_{\Lambda} v_{h}, \mu_{\odot h}\right\rangle_{\Lambda,|\partial \mathcal{D}|}}{\left\|v_{h}\right\|_{H^{1}(\Omega)}} \geq \beta_{h, 2}\left\|\mu_{\odot h}\right\|_{H^{-\frac{1}{2}}(\Lambda),|\partial \mathcal{D}|} \quad \forall \mu_{\odot h} \in Q_{h} .
$$

For any $w \in H^{\frac{1}{2}}(\Lambda)$, Lemma 2.1 ensures that $\left\|\mathcal{E}_{\Gamma} w\right\|_{H_{00}^{\frac{1}{2}}(\Gamma)}=\|w\|_{H_{00}^{\frac{1}{2}}(\Lambda),|\partial \mathcal{D}|}$. We use the extension operator $\mathcal{E}_{\Omega}^{h}$ from $X_{h, 0}^{k}(\Gamma)$ to $X_{h, 0}^{k}(\Omega)$, and we combine it with $\mathcal{E}_{\Gamma}^{h}$, namely the discrete uniform extension operator from $\Lambda$ to $\Gamma$ that for each node of $\mathcal{T}_{\mathfrak{h}}^{\Lambda}$ spans the nodal value of $q_{h} \in Q_{h}$ to the nodes of $\mathcal{T}_{h}^{\Omega}$ lying on the cross-section of $\Gamma$ that intersects the chosen node on $\mathcal{T}_{\mathfrak{h}}^{\Lambda}$ (see Figure 5.2 for a visualization). We call $\mathcal{E}_{\Omega}^{h} \mathcal{E}_{\Gamma}^{h}: Q_{h}:=X_{h, 0}^{k}(\Lambda) \rightarrow X_{h, 0}^{k}(\Omega)$ the combination of these two extensions. Through this construction, it is straightforward to see that $\overline{\mathcal{T}}_{\Lambda} \mathcal{E}_{\Omega}^{h} \mathcal{E}_{\Gamma}^{h}$ coincides with the identity operator on $Q_{h}$.

As a result, from Lemma 4.6, we obtain the following inequality:

$$
\begin{aligned}
\gamma_{h, 2}\left\|\mu_{\odot h}\right\|_{H^{-\frac{1}{2}}(\Lambda),|\partial \mathcal{D}|} & \leq \sup _{q_{h} \in Q_{h}} \frac{\left\langle q_{h}, \mu_{\odot h}\right\rangle_{\Lambda,|\partial \mathcal{D}|}}{\left\|q_{h}\right\|_{H_{00}^{\frac{1}{2}}(\Lambda),|\partial \mathcal{D}|}}=\sup _{q_{h} \in Q_{h}} \frac{\left\langle q_{h}, \mu_{\odot h}\right\rangle_{\Lambda,|\partial \mathcal{D}|}}{\left\|\mathcal{E}_{\Gamma}^{h} q_{h}\right\|_{H_{00}} \frac{1}{2}(\Gamma)} \\
& =C_{\mathcal{D}} \sup _{q_{h} \in Q_{h}} \frac{\left\langle\overline{\mathcal{T}}_{\Lambda} \mathcal{E}_{\Omega}^{h} \mathcal{E}_{\Gamma}^{h} q_{h}, \mu_{\odot h}\right\rangle_{\Lambda,|\partial \mathcal{D}|}}{\left\|\mathcal{E}_{\Omega}^{h} \mathcal{E}_{\Gamma}^{h} q_{h}\right\|_{H^{1}(\Omega)}} \\
& \leq C_{\mathcal{D}} \sup _{v_{h} \in X_{h, 0}^{h}} \frac{\left\langle\overline{\mathcal{T}}_{\Lambda} v_{h}, \mu_{\odot h}\right\rangle_{\Lambda,|\partial \mathcal{D}|}}{\left\|v_{h}\right\|_{H^{1}(\Omega)}} .
\end{aligned}
$$

This concludes the proof with $\beta_{h, 2}=\gamma_{h, 2} C_{\mathcal{D}}^{-1}$.

It is straightforward to see that problem (4.7a) satisfies properties equivalent to Corollary 4.5, with the only differences being that the Lagrange multiplier space is $X_{h, 0}^{k}(\Lambda)$ and that the approximation error of the Lagrange multiplier is measured in the norm of $\|\cdot\|_{H^{-\frac{1}{2}}(\Lambda),|\partial \mathcal{D}|}$. When $\mathcal{T}_{h}^{\Omega}$ conforms to $\mathcal{T}_{\mathfrak{h}}^{\Lambda}$ and to $\Gamma$, the discrete 3D-1D$2 \mathrm{D}$ and 3D-1D-1D problems may converge with optimal rates to the corresponding continuous problems, provided that the approximation error $\mathcal{E} \mathcal{R} \mathcal{R}\left(u, u_{\odot}, \lambda\right)$ features optimal properties. Such properties depend on the regularity of the solution $u, u_{\odot}, \lambda$. Assuming that such functions are poorly regular on the points of $\Gamma$ only, it is reasonable to expect that optimal convergence rates can be observed when the edges of the computational meshes resolve the surface $\Gamma$, for example, as in the conforming case. The numerical experiments shown in Table 5.1 provide good evidence of such behavior. However, we remark that this result is not interesting in practice, because the conformity assumptions require that $h \leq R_{0}$, where $R_{0}$ is the minimal cross-sectional radius of the inclusion. As a result, in this case the computational cost of the proposed scheme would be almost equivalent to the cost of resolving the full 3D-3D problem. To overcome this limitation, in the next section we develop an approximation method where $\mathcal{T}_{h}^{\Omega}$ and $\mathcal{T}_{\mathfrak{h}}^{\Lambda}$ do not conform to $\Gamma$.

4.2. Analysis of the case where $\mathcal{T}_{h}^{\Omega}$ and $\mathcal{T}_{\mathfrak{h}}^{\Lambda}$ do not conform to $\Gamma$. Now we analyze the case in which the elements of the $3 \mathrm{D}$ mesh $\mathcal{T}_{h}^{\Omega}$ do not conform with the surface $\Gamma$ or with $\Lambda$. As the 3D-1D-1D formulation is more suitable for this purpose, we focus solely on the analysis of the discrete version of problem 3D-1D-1D. 
4.2.1. Problem 3D-1D-1D. Let $u_{h} \in X_{h, 0}^{1}(\Omega)$ be the approximation of the $3 \mathrm{D}$ problem, and let $u_{\odot \mathfrak{h}} \in X_{\mathfrak{h}, 0}^{1}(\Lambda)$ be the approximation of the $1 \mathrm{D}$ problem. In contrast to the conforming case, here we limit the analysis to the case of piecewise linear finite elements. With slight abuse of notation, we use the subindex $h$ for the product space $X_{h}=X_{h, 0}^{1}(\Omega) \times X_{\mathfrak{h}, 0}^{1}(\Lambda)$. Concerning the multiplier space, let $\mathcal{G}_{h}=\left\{K \in \mathcal{T}_{h}^{\Omega}: K \cap \Lambda \neq \emptyset\right\}$ be the set of the $3 \mathrm{D}$ elements that intersect $\Lambda$. Then we define $Q_{h}=\left\{\lambda_{\odot h}: \lambda_{\odot h} \in P^{0}(K) \forall K \in \mathcal{G}_{h}\right\}$. We note that the multiplier functions are defined on the $3 \mathrm{D}$ elements. Again with slight abuse of notation, we denote by $Q_{h}$ also the restriction to $\Lambda$ of the space of piecewise constant functions defined in three dimensions. As a result, we have $Q_{h} \subset L^{2}(\Lambda) \subset H^{-\frac{1}{2}}(\Lambda)$. However, with this choice of multipliers, the problem is not inf-sup stable; therefore the idea is to add a stabilization term $s\left(\lambda_{\odot h}, \mu_{\odot h}\right): Q_{h} \times Q_{h} \rightarrow \mathbb{R}$ to (4.7a) following the approach introduced in [7].

The objective of this section is to analyze the following stabilized version of the 3D-1D-1D problem: find $\left[u_{h}, u_{\odot \mathfrak{h}}\right] \in X_{h}$ and $\lambda_{\odot h} \in Q_{h}$ such that

$$
\begin{aligned}
a\left(\left[u_{h}, u_{\odot \mathfrak{h}}\right],\left[v_{h}, v_{\odot \mathfrak{h}}\right]\right)+b\left(\left[v_{h}, v_{\odot \mathfrak{h}}\right], \lambda_{\odot h}\right)+b\left(\left[u_{h}, u_{\odot \mathfrak{h}}\right], \mu_{\odot h}\right) & \\
& -s_{h}\left(\lambda_{\odot h}, \mu_{\odot h}\right)=c\left(v_{h}\right)+d\left(\mu_{\odot h}\right) \quad \forall\left[v_{h}, v_{\odot \mathfrak{h}}\right] \in X_{h}, \forall \mu_{\odot h} \in Q_{h}
\end{aligned}
$$

The idea of the stabilization strategy proposed in [7] is to identify a new multiplier space $Q_{H}$, which is never implemented in practice, such that inf-sup stability with $X_{h}$ holds true. Then, the stabilization operator is designed to control the distance between $Q_{h}$ and $Q_{H}$ through the inequality

$$
\left\|\mu_{\odot h}-\pi_{H} \mu_{\odot h}\right\|_{Q_{H}} \leq C s_{h}\left(\mu_{\odot h}, \mu_{\odot h}\right)
$$

where $\pi_{H}$ is a suitable projection operator $Q_{h} \rightarrow Q_{H}$. Applying the results obtained in [7], the well-posedness of problem (4.9) is governed by the following lemma.

Lemma 4.8 (Lemma 2.3 of [7]). If the following assumptions are satisfied:

1. The bilinear form $b: X_{h} \times Q_{H} \rightarrow \mathbb{R}$ is inf-sup stable.

2. The stabilization operator $s_{h}: Q_{h} \times Q_{h} \rightarrow \mathbb{R}$ is such that

$$
\beta_{h}\left\|\mu_{\odot h}\right\|_{H^{-\frac{1}{2}}(\Lambda),|\partial \mathcal{D}|} \leq \sup _{v_{h} \in X_{h}} \frac{b\left(v_{h}, \mu_{\odot h}\right)}{\left\|v_{h}\right\|}+s_{h}\left(\mu_{\odot h}, \mu_{\odot h}\right) \quad \forall \mu_{\odot h} \in Q_{h},
$$

where $\beta_{h}$ is a positive constant independent of the mesh characteristic size.

3. For any $\left[v_{h}, v_{\odot \mathfrak{h}}\right] \in X_{h}$ there exists a function $\xi_{h} \in Q_{h}$ depending on $\left[v_{h}, v_{\odot \mathfrak{h}}\right]$, namely $\xi_{h}=\xi_{h}\left(\left[v_{h}, v_{\odot \mathfrak{h}}\right]\right)$, such that

$$
\begin{gathered}
a\left(\left[v_{h}, v_{\odot \mathfrak{h}}\right],\left[v_{h}, v_{\odot \mathfrak{h}}\right]\right)+b\left(\left[v_{h}, v_{\odot \mathfrak{h}}\right], \xi_{h}\right) \geq \alpha_{\xi}\left\|\left[v_{h}, v_{\odot \mathfrak{h}}\right]\right\|_{X_{h}}, \\
\left(s_{h}\left(\xi_{h}, \xi_{h}\right)\right)^{\frac{1}{2}} \leq c_{s}\left\|\left[v_{h}, v_{\odot \mathfrak{h}}\right]\right\| \|_{X_{h}},
\end{gathered}
$$

where $\|[\cdot, \cdot]\| \|_{X_{h}}$ is a suitable discrete norm.

Then, problem (4.9) admits a unique solution.

For the proof of this result we refer the reader to Lemma 2.3 of [7]. In the remainder of this section, we show how to find a multiplier space $Q_{H}$ and a stabilization operator $s_{h}$ such that all the assumptions of Lemma 4.8 are satisfied.

The first step consists of showing that there exists a discrete space $Q_{H}$ that satisfies the first assumption of Lemma 4.8. We recall that in the case of problem 
3D-1D-1D,

$$
b\left(\left[u_{h}, v_{\odot \mathfrak{h}}\right], \mu_{\odot h}\right)=\left(\overline{\mathcal{T}}_{\Lambda} v_{h}-v_{\odot \mathfrak{h}}, \mu_{\odot h}\right)_{\Lambda,|\partial \mathcal{D}|} .
$$

The construction of the inf-sup stable space $Q_{H}$ is based on macro elements of diameter $H$, where $H$ is sufficiently large. In particular, we assume that there exist positive constants $c_{h}$ and $c_{H}$ such that $c_{h} h \leq H \leq c_{H}^{-1} h$. The space is constructed assembling the $3 \mathrm{D}$ elements of $\mathcal{G}_{h}$ into macro patches $\omega_{j}$ such that $H \leq\left|\omega_{j} \cap \Lambda\right| \leq c H$ with $H=\min _{j}\left|\omega_{j} \cap \Lambda\right|$ and $c \geq 1$. Let $M_{j}$ be the number of elements of the patch $\omega_{j}$, namely, $\omega_{j}=\cup_{i=0}^{M_{j}} K_{i}$, where $K_{i} \in \mathcal{G}_{h}$. We assume that $M_{j}$ is uniformly bounded in $j$ by some $M \in \mathbb{N}$ and that the interiors of the patches $\omega_{j}$ are disjoint. We define $Q_{H}$ as the space of piecewise constant functions on the patches, namely $Q_{H}=\left\{\mu_{\odot H}: \mu_{\odot H} \in P^{0}\left(\omega_{j}\right) \forall j\right\}$. As previously pointed out for $Q_{h}$, we denote by $Q_{H}$ also the restriction of the multiplier space to $\Lambda$, namely $Q_{H} \subset L^{2}(\Lambda) \subset H^{-\frac{1}{2}}(\Lambda)$. Moreover, we associate to each patch $\omega_{j}$ a shape-regular extended patch (using the classical definition of shape-regularity; see, for example, [13]), still denoted by $\omega_{j}$ for notational simplicity, which is built by adding to $\omega_{j}$ a sufficient number of elements of $\mathcal{T}_{h}^{\Omega}$, and we assume that the interiors of the new extended patches $\omega_{j}$ are still disjoint (see Figure 4.1). The extended patches $\omega_{j}$ are built such that they fulfill the conditions meas $\left(\omega_{j}\right)=\mathcal{O}\left(H^{3}\right)$ and $\operatorname{diam}\left(\Gamma_{\omega_{j} \cap \Lambda} \cap \omega_{j}\right)=\mathcal{O}(H)(\mathcal{O}(X)$ means $c X \leq \mathcal{O}(X) \leq C X)$, where $\Gamma_{\omega_{j} \cap \Lambda}$ is the portion of $\Gamma$ with centerline $\omega_{j} \cap \Lambda$. The latter assumption is required to ensure that the intersection of $\Gamma_{\omega_{j} \cap \Lambda}$ and $\omega_{j}$ is not too small, and it will be needed later to prove the inf-sup stability of the space $Q_{H}$ in Lemma 4.9. A representation of this construction in the simple case in which $\omega_{j}$ is composed just by one tetrahedron is shown in Figure 4.1. Thanks to the shaperegularity of these extended patches, the following discrete trace inequality holds true for any function $v \in H^{1}\left(\omega_{j}\right)$ :

$$
\left\|\mathcal{T}_{\Gamma} v\right\|_{L^{2}\left(\Gamma \cap \omega_{j}\right)} \leq C_{I} H^{-\frac{1}{2}}\|v\|_{L^{2}\left(\omega_{j}\right)} .
$$

Moreover, $\forall u_{h} \in X_{h, 0}^{1}(\Omega)$ we have the following average inequality, which is a consequence of the definition of $\overline{\mathcal{T}}_{\Lambda}$, Jensen's inequality, and the fact that the patches are disjoint:

$$
\begin{aligned}
& \sum_{j}\left\|\overline{\mathcal{T}}_{\Lambda} u_{h}\right\|_{L^{2}\left(\omega_{j} \cap \Lambda\right),|\partial \mathcal{D}|}^{2}=\int_{\Lambda}|\partial \mathcal{D}|\left(\frac{1}{|\partial \mathcal{D}|} \int_{\partial \mathcal{D}} \mathcal{T}_{\Gamma} u_{h}\right)^{2} \\
& \leq \int_{\Lambda} \int_{\partial \mathcal{D}}\left(\mathcal{T}_{\Gamma} u_{h}\right)^{2}=\int_{\Gamma}\left(\mathcal{T}_{\Gamma} u_{h}\right)^{2}=\sum_{j} \int_{\omega_{j} \cap \Gamma}\left(\mathcal{T}_{\Gamma} u_{h}\right)^{2}=\sum_{j}\left\|\mathcal{T}_{\Gamma} u_{h}\right\|_{L^{2}\left(\omega_{j} \cap \Gamma\right)}^{2} .
\end{aligned}
$$

We are now ready to prove that the space $Q_{H}$ is inf-sup stable.

Lemma 4.9. The space $Q_{H}$ is inf-sup stable; namely, there exists $\beta_{H}>0$ independent of the characteristic size of macro patches such that

$$
\sup _{\substack{v_{h} \in X_{h, 0}^{1}(\Omega), v_{\odot \mathfrak{h}} \in X_{\mathfrak{h}, 0}^{1}(\Lambda)}} \frac{\left(\overline{\mathcal{T}}_{\Lambda} v_{h}-v_{\odot \mathfrak{h}}, \mu_{\odot H}\right)_{\Lambda,|\partial \mathcal{D}|}}{\left\|\left[v_{h}, v_{\odot \mathfrak{h}}\right]\right\| \mid} \geq \beta_{H}\left\|\mu_{\odot H}\right\|_{H^{-\frac{1}{2}}(\Lambda),|\partial \mathcal{D}|} \quad \forall \mu_{\odot H} \in Q_{H} .
$$



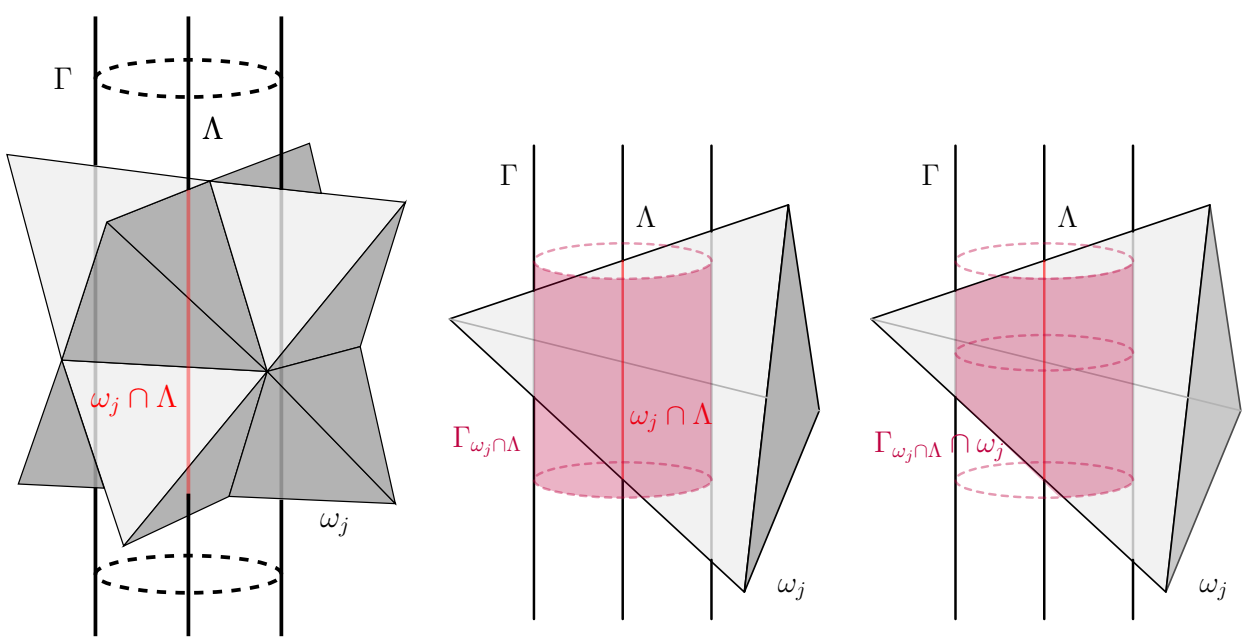

FIG. 4.1. (Left) Extended patches $\omega_{j}$. (Middle) $\Gamma_{\omega_{j} \cap \Lambda}$, the portion of $\Gamma$ generated by $\omega_{j} \cap \Lambda$. (Right) The intersection between $\Gamma_{\omega_{j} \cap \Lambda}$ and $\omega_{j}$. Here for simplicity $\omega_{j}$ is represented as a single tetrahedron, but actually it is a collection of tetrahedra as shown in the left panel.

Proof. We choose $v_{\odot \mathfrak{h}}=0$ and prove that

$$
\sup _{v_{h} \in X_{h, 0}^{1}(\Omega)} \frac{\left(\overline{\mathcal{T}}_{\Lambda} v_{h}, \mu_{\odot H}\right)_{\Lambda,|\partial \mathcal{D}|}}{\left\|v_{h}\right\|_{H^{1}(\Omega)}} \geq \beta_{H}\left\|\mu_{\odot H}\right\|_{H^{-\frac{1}{2}}(\Lambda),|\partial \mathcal{D}|} .
$$

Proving the last inequality is equivalent to finding the Fortin operator $\pi_{F}: H_{0}^{1}(\Omega) \rightarrow$ $X_{h, 0}^{1}(\Omega)$ such that

$$
\begin{gathered}
\left(\overline{\mathcal{T}}_{\Lambda} v-\overline{\mathcal{T}}_{\Lambda} \pi_{F} v, \mu_{\odot H}\right)_{\Lambda,|\partial \mathcal{D}|}=0 \quad \forall v \in H_{0}^{1}(\Omega), \mu_{\odot H} \in Q_{H} \\
\left\|\pi_{F} v\right\|_{H^{1}(\Omega)} \leq C\|v\|_{H^{1}(\Omega)} .
\end{gathered}
$$

We define

$$
\pi_{F} v=I_{h} v+\sum_{j} \alpha_{j} \varphi_{j} \quad \text { with } \alpha_{j}=\frac{\int_{\omega_{j} \cap \Lambda}|\partial \mathcal{D}|\left(\overline{\mathcal{T}}_{\Lambda} v-\overline{\mathcal{T}}_{\Lambda} I_{h} v\right)}{\int_{\omega_{j} \cap \Lambda}|\partial \mathcal{D}| \overline{\mathcal{T}}_{\Lambda} \varphi_{j}}
$$

where $I_{h}: H^{1}(\Omega) \rightarrow X_{h, 0}^{1}(\Omega)$ denotes an $H^{1}(\Omega)$-stable interpolant and $\varphi_{j} \in X_{h, 0}^{1}(\Omega)$ is such that $\operatorname{supp}\left(\varphi_{j}\right) \subset \omega_{j}, \operatorname{supp}\left(\mathcal{T}_{\Gamma} \varphi_{j}\right) \subset \Gamma_{\omega_{j} \cap \Lambda} \cap \omega_{j}, \varphi_{j}=0$ on $\partial \omega_{j}$, and

$$
\int_{\omega_{j} \cap \Lambda}|\partial \mathcal{D}| \overline{\mathcal{T}}_{\Lambda} \varphi_{j}=\mathcal{O}(H) \text { and }\left\|\nabla \varphi_{j}\right\|_{L^{2}\left(\omega_{j}\right)}=\mathcal{O}(1)
$$

We note that $\operatorname{supp}\left(\mathcal{T}_{\Gamma} \varphi_{j}\right) \subset \Gamma_{\omega_{j} \cap \Lambda} \cap \omega_{j}$ ensures that $\overline{\mathcal{T}}_{\Lambda} \varphi_{j} \subset \omega_{j} \cap \Lambda$. Therefore, since the interiors of $\omega_{j} \cap \Lambda$ are disjoint and $\varphi_{j}=0$ on $\partial \omega_{j}$, the functions $\overline{\mathcal{T}}_{\Lambda} \varphi_{j} \forall j$ all have disjoint supports. Provided $H$ is sufficiently larger than $h$, the functions $\varphi_{j}$ and their traces $\mathcal{T}_{\Gamma} \varphi_{j}$ have a sufficiently large support thanks to the facts that meas $\left(\omega_{j}\right)=\mathcal{O}\left(H^{3}\right)$ and $\operatorname{diam}\left(\Gamma_{\omega_{j} \cap \Lambda} \cap \omega_{j}\right)=\mathcal{O}(H)$. Owing to these properties, it is 
possible to satisfy (4.16). Then, by construction,

$$
\begin{gathered}
\left(\overline{\mathcal{T}}_{\Lambda} v-\overline{\mathcal{T}}_{\Lambda} \pi_{F} v, \mu_{\odot H}\right)_{\Lambda,|\partial \mathcal{D}|}=\sum_{j} \int_{\omega_{j} \cap \Lambda}|\partial \mathcal{D}|\left[\overline{\mathcal{T}}_{\Lambda} v-\overline{\mathcal{T}}_{\Lambda} I_{h} v-\sum_{i} \alpha_{i} \overline{\mathcal{T}}_{\Lambda} \varphi_{i}\right] \mu_{\odot H} \\
=\sum_{j} \int_{\omega_{j} \cap \Lambda}|\partial \mathcal{D}|\left[\overline{\mathcal{T}}_{\Lambda} v-\overline{\mathcal{T}}_{\Lambda} I_{h} v-\alpha_{j} \overline{\mathcal{T}}_{\Lambda} \varphi_{j}\right] \mu_{\odot H} \\
=\sum_{j}\left[\int_{\omega_{j} \cap \Lambda}|\partial \mathcal{D}|\left(\overline{\mathcal{T}}_{\Lambda} v-\overline{\mathcal{T}}_{\Lambda} I_{h} v\right) \mu_{\odot H}-\int_{\omega_{j} \cap \Lambda}|\partial \mathcal{D}|\left(\overline{\mathcal{T}}_{\Lambda} v-\overline{\mathcal{T}}_{\Lambda} I_{h} v\right) \mu_{\odot H}\right]=0 .
\end{gathered}
$$

Concerning the continuity of $\pi_{F}$, we exploit the assumption that the interiors of $\omega_{j}$ are disjoint, $\operatorname{supp}\left(\varphi_{j}\right) \subset \omega_{j}$, and the $H^{1}$-stability of $I_{h}$ to show that

$$
\left\|\nabla \pi_{F} v\right\|_{L^{2}(\Omega)} \leq C\|\nabla v\|_{L^{2}(\Omega)}+\left(\sum_{j} \alpha_{j}^{2}\left\|\nabla \varphi_{j}\right\|_{L^{2}\left(\omega_{j}\right)}^{2}\right)^{\frac{1}{2}} .
$$

For the second term, using the facts that $\left\|\nabla \varphi_{j}\right\|_{L^{2}\left(\omega_{j}\right)}=\mathcal{O}(1), \int_{\omega_{j} \cap \Lambda}|\partial \mathcal{D}| \overline{\mathcal{T}}_{\Lambda} \varphi_{j}=$ $\mathcal{O}(H)$ and that $\left|\omega_{j} \cap \Lambda\right| \leq c H$, exploiting Jensen's average inequality (4.13) and trace inequality (4.12), and finally applying the approximation properties of $I_{h}$, we see that the following upper bound holds true (where all the constants have been condensed into $C$ ):

$$
\begin{gathered}
\sum_{j} \alpha_{j}^{2}\left\|\nabla \varphi_{j}\right\|_{L^{2}\left(\omega_{j}\right)}^{2} \leq C \sum_{j} \frac{\left(\int_{\omega_{j} \cap \Lambda}|\partial \mathcal{D}|\left(\overline{\mathcal{T}}_{\Lambda} v-\overline{\mathcal{T}}_{\Lambda} I_{h} v\right)\right)^{2}}{\left(\int_{\omega_{j} \cap \Lambda}|\partial \mathcal{D}| \overline{\mathcal{T}}_{\Lambda} \varphi_{j}\right)^{2}} \\
\leq \frac{C}{H^{2}} \sum_{j}\left|\omega_{j} \cap \Lambda\right| \int_{\omega_{j} \cap \Lambda}|\partial \mathcal{D}|^{2}\left(\overline{\mathcal{T}}_{\Lambda} v-\overline{\mathcal{T}}_{\Lambda} I_{h} v\right)^{2} \\
\leq \frac{C}{H} \sum_{j}\left\|\overline{\mathcal{T}}_{\Lambda}\left(v-I_{h} v\right)\right\|_{L^{2}\left(\omega_{j} \cap \Lambda\right),|\partial \mathcal{D}|}^{2} \leq \frac{C}{H} \sum_{j}\left\|\mathcal{T}_{\Gamma}\left(v-I_{h} v\right)\right\|_{L^{2}\left(\omega_{j} \cap \Gamma\right)}^{2} \\
\leq \frac{C}{H^{2}} \sum_{j}\left\|v-I_{h} v\right\|_{L^{2}\left(\omega_{j}\right)}^{2} \leq C \frac{1}{H^{2}}\left\|v-I_{h} v\right\|_{L^{2}(\Omega)}^{2} \leq C\|\nabla v\|_{L^{2}(\Omega)}^{2},
\end{gathered}
$$

which is the $H^{1}$-stability of $\pi_{F}$. We notice that the constant in the inequality (4.15) is independent of how $\Lambda$ cuts the elements of the mesh $\mathcal{T}_{h}^{\Omega}$.

For the second assumption of Lemma 4.8, we recall that $b\left(v_{h}, \mu_{\odot h}\right)$ is continuous with respect to the norms $\left\|v_{h}\right\|,\left\|\mu_{\odot h}\right\|_{L^{2}(\Lambda)}$. Using Lemma 4.9 and, in particular, the existence of a Fortin projector, there exists a constant $\beta_{h}$ such that (the proof is analogous to that of Lemma 2.1 in [7])

$$
\beta_{h}\left\|\mu_{\odot h}\right\|_{H^{-\frac{1}{2}}(\Lambda),|\partial \mathcal{D}|} \leq \sup _{v_{h} \in X_{h}} \frac{b\left(v_{h}, \mu_{\odot h}\right)}{\left\|v_{h}\right\|}+\left\|\mu_{\odot h}-\pi_{H} \mu_{\odot h}\right\|_{L^{2}(\Lambda)} \quad \forall \mu_{\odot h} \in Q_{h} .
$$

We define $\pi_{H}=\sum_{j} \pi_{H}^{j}: L^{2}(\Lambda) \rightarrow Q_{H}$, where $\pi_{H}^{j}$ is the operator

$$
\pi_{H}^{j} w_{\mid \omega_{j} \cap \Lambda}=\frac{1}{\left|\Gamma_{\omega_{j} \cap \Lambda}\right|} \int_{\omega_{j} \cap \Lambda}|\partial \mathcal{D}| w \quad \forall j
$$

Copyright (c) by SIAM. Unauthorized reproduction of this article is prohibited. 
Since $\cup_{j} \omega_{j} \cap \Lambda=\Lambda$ and $\omega_{j} \cap \Lambda$ are not overlapping, we obtain that $\pi_{H}$ is an orthogonal projection, namely $\left(w-\pi_{H} w, \pi_{H} w\right)=0$. Moreover, for any $w \in L^{2}(\Lambda)$ the following Poincaré inequality holds true (see, for example, [13, Corollary B.65]):

$$
\left\|w-\pi_{H} w\right\|_{L^{2}\left(\omega_{j} \cap \Lambda\right),|\partial \mathcal{D}|} \leq C_{P} H\left\|\partial_{s} w\right\|_{L^{2}\left(\omega_{j} \cap \Lambda\right),|\partial \mathcal{D}|} .
$$

We consider the stabilization operator

$$
s_{h}\left(\lambda_{\odot h}, \mu_{\odot h}\right)=\sum_{K \in \mathcal{G}_{h}} \int_{\partial K \backslash \partial \mathcal{G}_{h}} h \llbracket \lambda_{\odot h} \rrbracket \llbracket \mu_{\odot h} \rrbracket,
$$

where $\llbracket \lambda_{\odot h} \rrbracket$ is the jump of $\lambda_{\odot h}$ across the internal faces of $\mathcal{G}_{h}$. Then, we use the result of $[7$, section III] to show that

$$
\left\|\mu_{\odot h}-\pi_{H} \mu_{\odot h}\right\|_{L^{2}(\Lambda)} \leq C s_{h}\left(\mu_{\odot h}, \mu_{\odot h}\right),
$$

which, combined with (4.17), shows that the second assumption of Lemma 4.8 holds true.

The third step of the analysis consists of showing that (4.10) and (4.11) are satisfied. We introduce the discrete norms

$$
\|\lambda\|_{ \pm \frac{1}{2}, h, \Lambda}=\left\|h^{\mp \frac{1}{2}} \lambda\right\|_{L^{2}(\Lambda)}
$$

and recall that $h$ is the mesh size of $\mathcal{T}_{h}^{\Omega}$. We equip the space $X_{h}$ with the discrete norm

$$
\left\|\left[u_{h}, u_{\odot \mathfrak{h}}\right]\right\|_{X_{h}}^{2}=\left\|u_{h}\right\|_{H^{1}(\Omega)}^{2}+\left\|u_{\odot \mathfrak{h}}\right\|_{H^{1}(\Lambda),|\mathcal{D}|}^{2}+\left\|\overline{\mathcal{T}}_{\Lambda} u_{h}-u_{\odot \mathfrak{h}}\right\|_{\frac{1}{2}, h, \Lambda,|\partial \mathcal{D}|}^{2}
$$

and furnish the space $Q_{H}$ with the $L^{2}$ norm $\left\|\mu_{\odot H}\right\|_{L^{2}(\Lambda)}$.

Also, the function $\xi_{h}\left(\left[v_{h}, v_{\odot \mathfrak{h}}\right]\right) \in Q_{H} \subset Q_{h} \subset L^{2}(\Lambda)$ is defined as

$$
\xi_{h \mid \omega_{j} \cap \Lambda}=\frac{\delta}{H} \pi_{H}\left(\overline{\mathcal{T}}_{\Lambda} u_{h}-u_{\odot \mathfrak{h}}\right)_{\mid \omega_{j} \cap \Lambda}
$$

where $\delta$ is an arbitrarily small parameter. Then the following result holds true.

Lemma 4.10. Given $\pi_{H}, s_{h}(\cdot, \cdot), \xi_{h}$ defined above and choosing $\delta$ small enough, we see that inequalities (4.10) and (4.11) are satisfied.

Proof. Concerning the coercivity property (4.10), we show that $\forall\left[u_{h}, u_{\odot \mathfrak{h}}\right]$, there exists $\xi_{h} \in Q_{h}$ such that

$$
\left(u_{h}, u_{h}\right)_{H^{1}(\Omega)}+\left(u_{\odot \mathfrak{h}}, u_{\odot \mathfrak{h}}\right)_{H^{1}(\Lambda),|\mathcal{D}|}+\left(\overline{\mathcal{T}}_{\Lambda} u_{h}-u_{\odot \mathfrak{h}}, \xi_{h}\right)_{\Lambda,|\partial \mathcal{D}|} \geq \alpha_{\xi}\left\|\left[u_{h}, u_{\odot \mathfrak{h}}\right]\right\|_{X_{h}}^{2} .
$$

Using the definitions of $\pi_{H}$ and $\xi_{h}\left(\left[u_{h}, u_{\odot \mathfrak{h}}\right]\right)$ previously presented and recalling that 
$\xi_{h} \in Q_{H} \subset Q_{h}$, we obtain

$$
\begin{gathered}
\left(\overline{\mathcal{T}}_{\Lambda} u_{h}-u_{\odot \mathfrak{h}}, \xi_{h}\right)_{\Lambda,|\partial \mathcal{D}|}=\frac{\delta}{H} \sum_{j} \pi_{H}^{j}\left(\overline{\mathcal{T}}_{\Lambda} u_{h}-u_{\odot \mathfrak{h}}\right) \int_{\omega_{j} \cap \Lambda}|\partial \mathcal{D}|\left(\overline{\mathcal{T}}_{\Lambda} u_{h}-u_{\odot \mathfrak{h}}\right) \\
=\frac{\delta}{H} \sum_{j} \int_{\omega_{j} \cap \Lambda}|\partial \mathcal{D}|\left(\pi_{H}\left(\overline{\mathcal{T}}_{\Lambda} u_{h}-u_{\odot \mathfrak{h}}\right)\right)^{2}=\frac{\delta}{H} \sum_{j}\left\|\pi_{H}\left(\overline{\mathcal{T}}_{\Lambda} u_{h}-u_{\odot \mathfrak{h}}\right)\right\|_{L^{2}\left(\omega_{j} \cap \Lambda\right),|\partial \mathcal{D}|}^{2} \\
=\frac{\delta}{H} \sum_{j}\left(\left\|\overline{\mathcal{T}}_{\Lambda} u_{h}-u_{\odot \mathfrak{h}}\right\|_{L^{2}\left(\omega_{j} \cap \Lambda\right),|\partial \mathcal{D}|}^{2}-\left\|\left(\pi_{H}-\mathcal{I}\right)\left(\overline{\mathcal{T}}_{\Lambda} u_{h}-u_{\odot \mathfrak{h}}\right)\right\|_{L^{2}\left(\omega_{j} \cap \Lambda\right),|\partial \mathcal{D}|}^{2}\right) \\
\geq \frac{\delta}{H} \sum_{j}\left(\left\|\overline{\mathcal{T}}_{\Lambda} u_{h}-u_{\odot \mathfrak{h}}\right\|_{L^{2}\left(\omega_{j} \cap \Lambda\right),|\partial \mathcal{D}|}^{2}-\left\|\left(\pi_{H}-\mathcal{I}\right) \overline{\mathcal{T}}_{\Lambda} u_{h}\right\|_{L^{2}\left(\omega_{j} \cap \Lambda\right),|\partial \mathcal{D}|}^{2}\right. \\
\left.-\left\|\left(\pi_{H}-\mathcal{I}\right) u_{\odot \mathfrak{h}}\right\|_{L^{2}\left(\omega_{j} \cap \Lambda\right),|\partial \mathcal{D}|}^{2}\right)
\end{gathered}
$$

Now, we seek an upper bound of the second and third (negative) terms of the last inequality. For the second term, we apply the additional assumption that the operators $\overline{\mathcal{T}}_{\Lambda}$ and $\partial_{s}$ commute. This is true if the cross-section $\mathcal{D}$ does not depend on the arclength $s$. Then, we use the Poincare inequality (4.19), the average inequality (4.13), and the trace inequality (4.12) to show that

$$
\begin{aligned}
\sum_{j}\left\|\left(\pi_{H}-\mathcal{I}\right) \overline{\mathcal{T}}_{\Lambda} u_{h}\right\|_{L^{2}\left(\omega_{j} \cap \Lambda\right),|\partial \mathcal{D}|}^{2} \leq C_{P}^{2} H^{2} \sum_{j}\left\|\overline{\mathcal{T}}_{\Lambda} \partial_{s} u_{h}\right\|_{L^{2}\left(\omega_{j} \cap \Lambda\right),|\partial \mathcal{D}|}^{2} \\
\leq C_{P}^{2} H^{2} \sum_{j}\left\|\mathcal{T}_{\Gamma} \partial_{s} u_{h}\right\|_{L^{2}\left(\omega_{j} \cap \Gamma\right)}^{2} \leq C_{P}^{2} C_{I}^{2} H \sum_{j}\left\|\nabla u_{h}\right\|_{L^{2}\left(\omega_{j}\right)}^{2} .
\end{aligned}
$$

For the third term, the following upper bound holds true:

$$
\begin{aligned}
\sum_{j}\left\|\left(\pi_{H}-\mathcal{I}\right) u_{\odot \mathfrak{h}}\right\|_{L^{2}\left(\omega_{j} \cap \Lambda\right),|\partial \mathcal{D}|}^{2} \leq C_{P}^{2} & H^{2} \sum_{j}\left\|\partial_{s} u_{\odot \mathfrak{h}}\right\|_{L^{2}\left(\omega_{j} \cap \Lambda\right),|\partial \mathcal{D}|}^{2} \\
& \leq C_{P}^{2} H^{2} \frac{\max |\partial \mathcal{D}|}{\min |\mathcal{D}|} \sum_{j}\left\|\partial_{s} u_{\odot \mathfrak{h}}\right\|_{L^{2}\left(\omega_{j} \cap \Lambda\right),|\mathcal{D}|}^{2} .
\end{aligned}
$$

Combining the last three inequalities and recalling that $c_{h} h \leq H \leq c_{H}^{-1} h$, we obtain

$$
\begin{aligned}
& a\left(\left[u_{h}, u_{\odot \mathfrak{h}}\right],\left[u_{h}, u_{\odot \mathfrak{h}}\right]\right)+b\left(\left[u_{h}, u_{\odot \mathfrak{h}}\right], \xi_{h}\left(\left[u_{h}, u_{\odot \mathfrak{h}}\right]\right)\right) \geq\left(1-\delta C_{P}^{2} C_{I}^{2}\right)\left\|\nabla u_{h}\right\|_{L^{2}(\Omega)}^{2} \\
& \quad+\left(1-\delta C_{P}^{2} H \frac{\max |\partial \mathcal{D}|}{\min |\mathcal{D}|}\right)\left\|\partial_{s} u_{\odot \mathfrak{h}}\right\|_{L^{2}(\Lambda),|\mathcal{D}|}^{2}+\delta c_{H}\left\|\overline{\mathcal{T}}_{\Lambda} u_{h}-u_{\odot \mathfrak{h}}\right\|_{\frac{1}{2}, h, \Lambda,|\partial \mathcal{D}|}^{2},
\end{aligned}
$$

and choosing

$$
\delta=\frac{1}{2} \min \left[\left(C_{P}^{2} C_{I}^{2}\right)^{-1},\left(C_{P}^{2} H \frac{\max |\partial \mathcal{D}|}{\min |\mathcal{D}|}\right)^{-1}\right],
$$

we obtain the desired inequality. The proof of (4.11) is analogous to that in [7].

5. A benchmark problem with analytical solution. Let $\Omega=[0,1]^{3}, \Lambda=$ $\left\{x=\frac{1}{2}\right\} \times\left\{y=\frac{1}{2}\right\} \times[0,1]$, and $\Omega_{\ominus}=\left[\frac{1}{4}, \frac{3}{4}\right] \times\left[\frac{1}{4}, \frac{3}{4}\right] \times[0,1]$. As a benchmark for the two formulations (2.4) and (2.3), we consider the case in which the source terms are defined as

$$
f=8 \pi^{2} \sin (2 \pi x) \sin (2 \pi y), \quad \overline{\bar{g}}=\pi^{2} \sin (\pi z),
$$

Copyright $\odot$ by SIAM. Unauthorized reproduction of this article is prohibited. 
and $q_{1}$ for $(2.4)$ and $\bar{q}_{2}$ for $(2.3)$ are given by

$$
q_{1}=\sin (2 \pi x) \sin (2 \pi y)-\sin (\pi z), \quad \bar{q}_{2}=-\sin (\pi z) .
$$

At the boundary $\partial \Omega$, the following nonhomogeneous Dirichlet conditions are imposed:

$$
u=u_{b} \text { on } \partial \Omega \quad \text { with } u_{b}=\sin (2 \pi x) \sin (2 \pi y) .
$$

Under these conditions, the solutions of (2.4) and (2.3) are given by

$$
u=\sin (2 \pi x) \sin (2 \pi y), \quad u_{\odot}=\sin (\pi z), \quad \lambda=\lambda_{\odot}=0 .
$$

We show that (5.1) is the solution of (2.3). We notice that, regardless of the coupling constraints, $u$ and $u_{\odot}$ are solutions of the following problem:

$$
\begin{aligned}
-\Delta u=f & \text { in } \Omega, \\
-d_{z z}^{2} u_{\odot}=\overline{\bar{g}} & \text { on } \Lambda, \\
u=u_{b} & \text { on } \partial \Omega .
\end{aligned}
$$

Using the integration-by-parts formula and homogeneous boundary conditions on $\Omega$ and $\Lambda$, from (2.3) we have

$$
\begin{aligned}
& -(\Delta u, v)_{L^{2}(\Omega)}-|\mathcal{D}|\left(d_{s s}^{2} u_{\odot}, v_{\odot}\right)_{L^{2}(\Lambda)}+|\mathcal{D}|\left\langle\bar{v}-v_{\odot}, \lambda_{\odot}\right\rangle_{\Lambda} \\
& \quad=(f, v)_{L^{2}(\Omega)}+|\mathcal{D}|\left(\overline{\bar{g}}, v_{\odot}\right)_{L^{2}(\Lambda)} \quad \forall v \in H_{0}^{1}(\Omega), v_{\odot} \in H_{0}^{1}(\Lambda) .
\end{aligned}
$$

Since $\lambda_{\odot}=0$ and the first term of (5.1) satisfies (5.2a) and the second satisfies (5.2b), we have that

$$
\begin{array}{r}
-(\Delta u, v)_{L^{2}(\Omega)}=(f, v)_{L^{2}(\Omega)}, \\
-|\mathcal{D}|\left(d_{s s}^{2} u_{\odot}, v_{\odot}\right)_{L^{2}(\Lambda)}=|\mathcal{D}|\left(\overline{\bar{g}}, v_{\odot}\right)_{L^{2}(\Lambda)} .
\end{array}
$$

Thus (5.1) satisfies (2.3a) and (2.3b). The fact that the solution satisfies (2.3c) follows from (5.1) and the definition of $\bar{q}_{2}$.

We can prove in a similar way that (5.1) satisfies (2.4). Note in particular that $q_{1}$ is such that $\mathcal{T}_{\Gamma} u-\mathcal{E}_{\Gamma} u_{\odot}=q_{1}$ on $\Gamma$.

5.1. Numerical experiments. $\mathcal{T}_{h}^{\Omega}$ conforming to $\Gamma$. Using the benchmark solution (5.1), we now investigate convergence properties of the two formulations. To this end we consider a uniform mesh of $\mathcal{T}_{h}^{\Omega}$ of $\Omega$ consisting of tetrahedra with diameter $h$. Further, the discretization shall be geometrically conforming to both $\Lambda$ and $\Gamma$ such that the meshes $\mathcal{T}_{h}^{\Gamma}, \mathcal{T}_{h}^{\Lambda}$ are made up of facets and edges of $\mathcal{T}_{h}^{\Omega}$; cf. Figure 5.1 for illustration.

Considering inf-sup stable discretization in terms of continuous linear Lagrange $\left(P_{1}\right)$ elements (for all the spaces), Table 5.1 lists the errors of formulations (2.4) and (2.3) on the benchmark problem. It can be seen that the error in $u$ and $u_{\odot}$ in the $H^{1}$ norm converges linearly (as can be expected due to $P_{1}$ element discretization). Moreover, the error of the Lagrange multiplier approximation in the $H^{-1 / 2}$ norm decreases quadratically. In light of $P_{1}$ discretization, this rate appears superconvergent. We speculate that the result is due to the fact that the exact solution is particularly simple, that is, $\lambda=\lambda_{\odot}=0$. We remark that for $u$ and $u_{\odot}$ the error is interpolated 

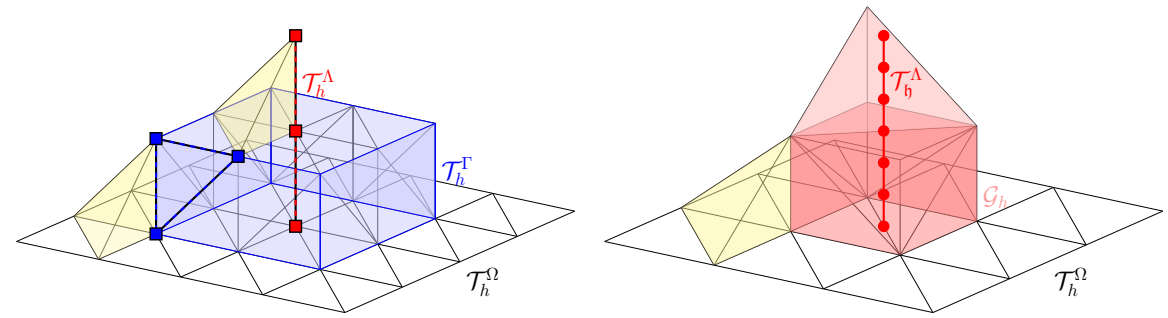

FIG. 5.1. (Left) The conforming discretization of $\Lambda, \Gamma$, and $\Omega$ used for (2.4) and (2.3) is highlighted. Each cell of $\mathcal{T}_{h}^{\Gamma}$ (in blue, filled marker vertices) and $\mathcal{T}_{h}^{\Lambda}$ (in red, filled marker vertices) is a facet (respectively, edge) of $\mathcal{T}_{h}^{\Omega}$ (in black, empty square marker vertices). (Right) Sample discretization of the benchmark geometry in the nonconforming case for (2.3). (See online version for color.)

TABLE 5.1

Error convergence on a benchmark problem (5.2). (Top) problem (2.4), (middle) (2.3) with conforming discretization, and (bottom) (2.3) in case $\mathcal{T}_{h}^{\Omega}$ does not conform to $\Lambda$ using stabilized formulation (4.9). Continuous linear Lagrange elements are used for $u_{h}, u_{\odot h}$, and $u_{\odot \mathfrak{h}}$, and for $\lambda_{\odot h}$ in the conforming case, while in the nonconforming case, $\lambda_{\odot h}$ is piecewise constant on elements of $\mathcal{G}_{h}$.

\begin{tabular}{l|llll}
\hline \multicolumn{5}{c}{$\mathcal{T}_{h}^{\Omega}$ conforming to $\Gamma, \Lambda$} \\
\hline$h^{-1}$ & $\left\|u-u_{h}\right\|_{H^{1}(\Omega)}$ & $\left\|u_{\odot}-u_{\odot h}\right\|_{H^{1}(\Lambda)}$ & $\left\|\lambda-\lambda_{h}\right\|_{H^{-1 / 2}(\Gamma)}$ & $\left\|\lambda-\lambda_{h}\right\|_{L^{2}(\Gamma)}$ \\
\hline 4 & $3.4 \mathrm{E} 0(-)$ & $5.3 \mathrm{E}-1(-)$ & $2.9 \mathrm{E} 0(-)$ & $8.7 \mathrm{E} 0(-)$ \\
8 & $1.7 \mathrm{E} 0(0.99)$ & $2.6 \mathrm{E}-1(1.06)$ & $6.1 \mathrm{E}-1(2.25)$ & $1.9 \mathrm{E} 0(2.21)$ \\
16 & $8.7 \mathrm{E}-1(0.99)$ & $1.3 \mathrm{E}-1(1.02)$ & $1.4 \mathrm{E}-1(2.13)$ & $4.7 \mathrm{E}-1(1.99)$ \\
32 & $4.4 \mathrm{E}-1(1.00)$ & $6.3 \mathrm{E}-2(1.00)$ & $3.4 \mathrm{E}-2(2.03)$ & $1.3 \mathrm{E}-1(1.80)$ \\
64 & $2.2 \mathrm{E}-1(1.00)$ & $3.1 \mathrm{E}-2(1.00)$ & $8.6 \mathrm{E}-3(2.00)$ & $4.2 \mathrm{E}-2(1.68)$ \\
\hline$h^{-1}$ & $\left\|u-u_{h}\right\|_{H^{1}(\Omega)}$ & $\left\|u_{\odot}-u_{\odot}\right\|_{H^{1}(\Lambda)}$ & $\left\|\lambda_{\odot}-\lambda_{\odot h}\right\|_{H^{-1 / 2}(\Lambda)}$ & $\left\|\lambda_{\odot}-\lambda_{\odot h}\right\|_{L^{2}(\Lambda)}$ \\
\hline 4 & $3.1 \mathrm{E} 0(-)$ & $5.4 \mathrm{E}-1(-)$ & $4.4 \mathrm{E}-2(-)$ & $7.8 \mathrm{E}-2(-)$ \\
8 & $1.7 \mathrm{E} 0(0.87)$ & $2.6 \mathrm{E}-1(1.06)$ & $1.1 \mathrm{E}-2(2.01)$ & $1.9 \mathrm{E}-2(2.01)$ \\
16 & $8.6 \mathrm{E}-1(0.96)$ & $1.3 \mathrm{E}-1(1.02)$ & $2.7 \mathrm{E}-3(2.01)$ & $4.8 \mathrm{E}-3(2.02)$ \\
32 & $4.4 \mathrm{E}-1(0.99)$ & $6.3 \mathrm{E}-2(1.00)$ & $6.7 \mathrm{E}-4(2.01)$ & $1.2 \mathrm{E}-3(2.01)$ \\
64 & $2.2 \mathrm{E}-1(1.00)$ & $3.1 \mathrm{E}-2(1.00)$ & $1.7 \mathrm{E}-4(2.01)$ & $3.0 \mathrm{E}-4(2.01)$ \\
128 & $1.1 \mathrm{E}-1(1.00)$ & $1.6 \mathrm{E}-2(1.00)$ & $4.1 \mathrm{E}-5(2.01)$ & $7.4 \mathrm{E}-5(2.00)$ \\
\hline \multicolumn{5}{c}{$\mathcal{T}_{h}^{\Omega}$ nonconforming to $\Gamma, \Lambda$} \\
\hline$h^{-1}$ & $\left.\left\|u-u_{h}\right\|_{H^{1}(\Omega)}\right)$ & $\left\|u_{\odot}-u_{\odot \mathfrak{h}}\right\|_{H^{1}(\Lambda)}$ & $\left\|\lambda_{\odot}-\lambda_{\odot h}\right\|_{L^{2}\left(\mathcal{G}_{h}\right)}$ \\
\hline 5 & $2.6 \mathrm{E} 0(-)$ & $2.3 \mathrm{E}-1(-)$ & $1.7 \mathrm{E}-1(-)$ \\
9 & $1.5 \mathrm{E} 0(0.84)$ & $9.4 \mathrm{E}-2(1.42)$ & $7.1 \mathrm{E}-2(1.36)$ \\
17 & $8.1 \mathrm{E}-1(0.94)$ & $4.3 \mathrm{E}-2(1.18)$ & $2.9 \mathrm{E}-2(1.37)$ \\
33 & $4.2 \mathrm{E}-1(0.98)$ & $2.1 \mathrm{E}-2(1.06)$ & $7.9 \mathrm{E}-3(1.91)$ \\
65 & $2.1 \mathrm{E}-1(0.99)$ & $1.1 \mathrm{E}-2(1.02)$ & $2.6 \mathrm{E}-3(1.64)$ \\
129 & $1.1 \mathrm{E}-1(1.00)$ & $5.2 \mathrm{E}-3(1.01)$ & $8.5 \mathrm{E}-4(1.61)$ \\
\hline \multicolumn{5}{l}{}
\end{tabular}

into the finite element space of piecewise quadratic discontinuous functions. For (2.3) we evaluate the fractional norm and interpolate the error using piecewise continuous cubic functions. For the sake of comparison with nonconforming formulation of (2.3) from section 4.2, Table 5.1 also lists the error of the Lagrange multiplier in the $L^{2}$ norm. Here, quadratic convergence is observed for (2.3). For (2.4) the rate is between 1.5 and 2.

We plot the numerical solution of problems (2.4) and (2.3) in Figure 5.2.

5.2. Numerical experiments. $\mathcal{T}_{h}^{\Omega}$ nonconforming to $\Gamma$. Using the proposed benchmark problem, we consider (2.3) in the setting of section 4.2. To this end we let $\mathcal{T}_{h}^{\Omega}$ be a uniform mesh of $\Omega$ such that no cell $\mathcal{T}_{h}^{\Omega}$ has any edge lying on $\Lambda$. 

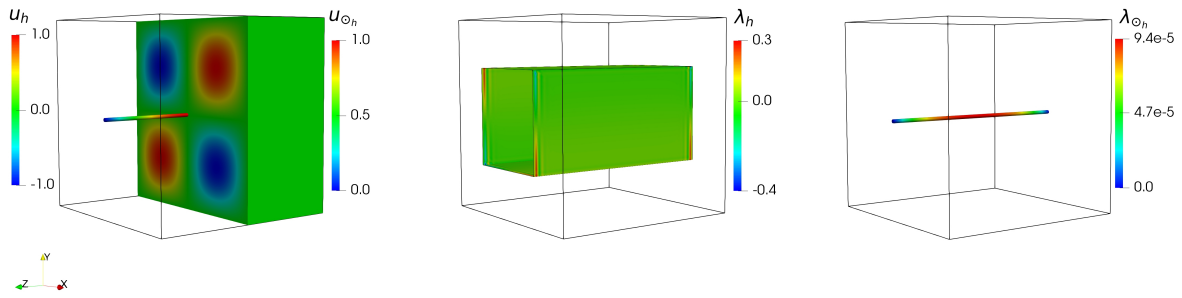

FIG. 5.2. Numerical solution of problems (2.4) and (2.3). (Left) Functions $u_{h}, u_{\odot h}$ (practically identical in both problems). (Middle) Lagrange multiplier for (2.4) and (right) for (2.3).

Further, we let $\mathfrak{h}=h / 3$ in $\mathcal{T}_{\mathfrak{h}}^{\Lambda}$; cf. Figure 5.1.

Using discretization in terms of the $P_{1}-P_{1}-P_{0}$ element, Table 5.1 lists the error of the stabilized formulation of (2.3). A linear convergence in the $H^{1}$ norm can be observed in the error of $u$ and $u_{\odot}$. We remark that the norms were computed as in section 5.1. For simplicity the convergence of the multiplier is measured in the $L^{2}$ norm rather than the $H^{-1 / 2}(\Gamma)$ norm used in the analysis. Then, convergence exceeding order 1.5 can be observed; however, the rates are rather unstable.

5.3. Comparison. In Table 5.1 one can observe that all the formulations yield practically identically accurate approximations of $u$. Further, compared to the conforming case, the stabilized formulation (2.3) results in a greater accuracy of $u_{\odot h}$ as the underlying mesh $\mathcal{T}_{h}^{\Lambda}$ is here finer. Due to the different definitions in the three formulations, comparison of the Lagrange multiplier convergence is not straightforward. We therefore limit ourselves to commenting that in the $L^{2}$ norm all the formulations yield faster-than-linear convergence. In order to discuss solution cost of the formulations we consider the resulting preconditioned linear systems. In particular, we shall compare spectral condition numbers and the time to convergence of the preconditioned minimal residual (MinRes) solver with the stopping criterion, and we require the relative preconditioned residual norm to be less than $10^{-8}$. We remark that we shall ignore the setup cost of the preconditioner. Following the operator preconditioning technique of [25], we propose as preconditioners for (2.4) and (2.3) in the conforming case the (approximate) Riesz mapping with respect to the inner products of the spaces in which the two formulations were proved to be well-posed. In particular, the preconditioner for the Lagrange multiplier relies on (the inverse of) the fractional Laplacian $-\Delta^{-1 / 2}$ on $\Gamma$ for (2.4) and $\Lambda$ for (2.3). A detailed analysis of the preconditioners will be presented in a separate work. We remark that in both cases the fractional was realized here by spectral decomposition [22]. For the unfitted stabilized formulation (2.3) the Lagrange multiplier preconditioner uses a Riesz map with respect to the inner product due to $L^{2}\left(\mathcal{G}_{h}\right)$ and the stabilization (4.20), i.e.,

$$
\left(\lambda_{\odot h}, \mu_{\odot}\right) \mapsto \sum_{K \in \mathcal{G}_{h}} \int_{K} \lambda_{\odot h} \mu_{\odot h}+\sum_{K \in \mathcal{G}_{h}} \int_{\partial K \backslash \partial \mathcal{G}_{h}} h \llbracket \lambda_{\odot h} \rrbracket \llbracket \mu_{\odot h} \rrbracket .
$$

This simple choice does not yield bounded iterations. However, establishing a robust preconditioner in this case is beyond the scope of this paper and shall be pursued in future work. In Table 5.2 we compare solution time, number of iterations, and condition numbers of the (linear systems due to the) three formulations. Let us first note that the proposed preconditioners for (2.4) and (2.3) in the conforming case seem robust with respect to the discretization parameter as the iteration counts and condition numbers are bounded in $h$. We then see that the solution time for (2.4) is about 
TABLE 5.2

Cost comparison of the formulations across refinement levels $l$. The number of Krylov iterations (preconditioned conjugate gradient for (5.2), MinRes otherwise) and the condition number of the preconditioned problem are denoted by \# and $\kappa$, respectively. Time until convergence of the iterative solver (excluding the setup) is shown as $T$.

\begin{tabular}{l|lll|lll|lll|ll|}
\hline \multirow{2}{*}{$l$} & \multicolumn{3}{|c|}{$(2.4)$} & \multicolumn{4}{|c|}{$(2.3)$} & \multicolumn{3}{c|}{ Stabilized $(2.3)$} & \multicolumn{2}{c}{$(5.2)$} \\
\cline { 2 - 22 } & $\#$ & $T[s]$ & $\kappa$ & $\#$ & $T[s]$ & $\kappa$ & $\#$ & $T[s]$ & $\kappa$ & $\#$ & $T s]$ \\
\hline 1 & 20 & 0.03 & 15.56 & 9 & 0.02 & 3.79 & 21 & 0.01 & 9.70 & 3 & $<0.01$ \\
2 & 35 & 0.06 & 16.28 & 17 & 0.03 & 6.04 & 31 & 0.03 & 15.87 & 4 & $<0.01$ \\
3 & 38 & 0.14 & 16.64 & 22 & 0.06 & 8.28 & 53 & 0.15 & 32.93 & 5 & 0.01 \\
4 & 39 & 1.70 & 16.75 & 24 & 0.89 & 9.42 & 110 & 4.54 & 61.48 & 5 & 0.12 \\
5 & 38 & 12.04 & 16.78 & 20 & 5.21 & 6.52 & 232 & 59.43 & 94.25 & 5 & 0.90 \\
6 & - & - & - & 17 & 28.77 & - & 507 & 832.90 & - & 6 & 7.75 \\
\hline
\end{tabular}

two times longer compared to that for (2.3) which is about four times more expensive than the solution of the Poisson problem (5.2) (which does not include any coupling, i.e., solved only for $u$ and $u_{\odot}$ ). This is in addition to the higher setup cost of the preconditioner, which in our implementation involves solving an eigenvalue problem for the fractional Laplacian. Therefore it is advantageous to keep the multiplier space as small as possible. We remark that the missing results for (2.4) in Table 5.2 are due to the memory limitations encountered when solving the eigenvalue problem for the Laplacian, which for finest mesh involves approximately 32,000 eigenvalues; cf. section $\mathrm{SM} 3$ of the supplementary material. Due to the missing proper preconditioner for the Lagrange multiplier block, the number of iterations in the third, unfitted formulation can be seen to approximately double on refinement.

\section{REFERENCES}

[1] R. A. Adams, Sobolev Spaces, Pure and Applied Mathematics 65, Academic Press, 1975.

[2] M. ARioli And D. Loghin, Discrete interpolation norms with applications, SIAM J. Numer. Anal., 47 (2009), pp. 2924-2951, https://doi.org/10.1137/080729360.

[3] M. Berg, Y. Davit, M. Quintard, and S. Lorthois, Modelling solute transport in the brain microcirculation: Is it really well mixed inside the blood vessels?, J. Fluid Mech., 884 (2020), A39.

[4] S. Bertoluzza, A. Decoene, L. Lacouture, and S. Martin, Local error estimates of the finite element method for an elliptic problem with a Dirac source term, Numer. Methods Partial Differential Equations, 34 (2018), pp. 97-120.

[5] W. Boon, J. Nordbotten, and J. Vatne, Mixed-Dimensional Elliptic Partial Differential Equations, preprint, https://arxiv.org/abs/1710.00556v2, 2017.

[6] F. BrEzzI, On the existence, uniqueness and approximation of saddle-point problems arising from Lagrangian multipliers, Rev. Française Automat. Informat. Recherche Opérationnelle Sér. Rouge, 8 (1974), pp. 129-151.

[7] E. Burman, Projection stabilization of Lagrange multipliers for the imposition of constraints on interfaces and boundaries, Numer. Methods for Partial Differential Equations, 30 (2014), pp. 567-592.

[8] D. Cerroni, F. Laurino, And P. Zunino, Mathematical analysis, finite element approximation and numerical solvers for the interaction of $3 D$ reservoirs with $1 D$ wells, GEM Int. J. Geomath., 10 (2019), 4.

[9] S. N. Chandler-Wilde, D. P. Hewett, and A. Moiola, Interpolation of Hilbert and Sobolev spaces: Quantitative estimates and counterexamples, Mathematika, 61 (2015), pp. 414-443.

[10] C. D'Angelo, Multiscale Modelling of Metabolism and Transport Phenomena in Living Tissues, Ph.D. thesis, EPFL, Lausanne, 2007.

[11] C. D'Angelo, Finite element approximation of elliptic problems with Dirac measure terms in weighted spaces: Applications to one-and three-dimensional coupled problems, SIAM J. Numer. Anal., 50 (2012), pp. 194-215, https://doi.org/10.1137/100813853.

[12] C. D'ANGelo AND A. QuARTERONI, On the coupling of $1 D$ and $3 D$ diffusion-reaction equations: Application to tissue perfusion problems, Math. Models Methods Appl. Sci., 18 (2008), pp. 1481-1504.

Copyright (C) by SIAM. Unauthorized reproduction of this article is prohibited. 
[13] A. Ern and J.-L. Guermond, Theory and Practice of Finite Elements, Appl. Math. Sci. 159, Springer-Verlag, 2004.

[14] Q. Fang, S. Sakadžić, L. Ruvinskaya, A. Devor, A. M. Dale, and D. A. Boas, Oxygen advection and diffusion in a three dimensional vascular anatomical network, Opt. Express, 16 (2008), pp. 17530-17541.

[15] I. Gansca, W. F. Bronsvoort, G. Coman, and L. Tambulea, Self-intersection avoidance and integral properties of generalized cylinders, Comput. Aided Geom. Design, 19 (2002), pp. 695-707.

[16] I. G. Gjerde, K. Kumar, and J. M. Nordbotten, A singularity removal method for coupled 1D-3D flow models, Comput. Geosci., 24 (2020), pp. 443-457.

[17] I. G. Gould, P. Tsai, D. Kleinfeld, And A. Linninger, The capillary bed offers the largest hemodynamic resistance to the cortical blood supply, J. Cerebral Blood Flow Metabolism, 37 (2017), pp. 52-68.

[18] T. Köppl, E. Vidotto, And B. Wohlmuth, A local error estimate for the Poisson equation with a line source term, in Numerical Mathematics and Advanced Applications (ENUMATH 2015), Springer, 2016, pp. 421-429.

[19] T. Köppl, E. Vidotto, B. Wohlmuth, And P. Zunino, Mathematical modeling, analysis and numerical approximation of second-order elliptic problems with inclusions, Math. Models Methods Appl. Sci., 28 (2018), pp. 953-978.

[20] T. KöPpl AND B. Wohlmuth, Optimal a priori error estimates for an elliptic problem with Dirac right-hand side, SIAM J. Numer. Anal., 52 (2014), pp. 1753-1769, https://doi.org/ $10.1137 / 130927619$.

[21] M. Kuchta, K.-A. Mardal, and M. Mortensen, Preconditioning trace coupled $3 d-1 d$ systems using fractional Laplacian, Numer. Methods Partial Differential Equations, 35 (2019), pp. 375-393.

[22] M. Kuchta, M. Nordaas, J. C. G. Verschaeve, M. Mortensen, and K.-A. Mardal, Preconditioners for saddle point systems with trace constraints coupling $2 D$ and $1 D$ domains, SIAM J. Sci. Comput., 38 (2016), pp. B962-B987, https://doi.org/10.1137/15M1052822.

[23] F. LAURino And P. Zunino, Derivation and analysis of coupled PDEs on manifolds with high dimensionality gap arising from topological model reduction, ESAIM Math. Model. Numer. Anal., 53 (2019), pp. 2047-2080.

[24] J.-L. Lions And E. Magenes, Non-homogeneous Boundary Value Problems and Applications. Vol. I, translated from the French by P. Kenneth, Grundlehren Math. Wiss. 181, SpringerVerlag, 1972.

[25] K.-A. Mardal and R. Winther, Preconditioning discretizations of systems of partial differential equations, Numer. Linear Algebra Appl., 18 (2011), pp. 1-40.

[26] Y. Mori, A. Rodenberg, And D. SpIRn, Well-posedness and global behavior of the Peskin problem of an immersed elastic filament in Stokes flow, Commun. Pure Appl. Math., 72 (2019), pp. 887-980.

[27] D. Peaceman, Interpretation of well-block pressures in numerical reservoir simulation, Soc. Pet. Eng. AIME J., 18 (1978), pp. 183-194.

[28] D. W. Peaceman, Interpretation of well-block pressures in numerical reservoir simulation with nonsquare grid blocks and anisotropic permeability, Soc. Pet. Eng. J., 23 (1983), pp. 531543.

[29] L. Possenti, G. Casagrande, S. Di Gregorio, P. Zunino, and M. Costantino, Numerical simulations of the microvascular fluid balance with a non-linear model of the lymphatic system, Microvascular Res., 122 (2019), pp. 101-110.

[30] S. SAUter AND R. WARNKe, Extension operators and approximation on domains containing small geometric details, East West J. Numer. Math., 7 (1999), pp. 61-77.

[31] L. R. ScotT And S. Zhang, Finite element interpolation of nonsmooth functions satisfying boundary conditions, Math. Comp., 54 (1990), pp. 483-493.

[32] T. W. Secomb, R. Hsu, E. Y. Park, and M. W. Dewhirst, Green's function methods for analysis of oxygen delivery to tissue by microvascular networks, Ann. Biomed. Engrg., 32 (2004), pp. 1519-1529.

[33] V. Vinje, G. Ringstad, E. Lindstrøm, L. Valnes, M. Rognes, P. Eide, and K.-A. Mardal, Respiratory influence on cerebrospinal fluid flow-a computational study based on long-term intracranial pressure measurements, Sci. Rep., 9 (2019), 9732.

[34] O. B. Widuund, An Extension Theorem for Finite Element Spaces with Three Applications, Vieweg+Teubner Verlag, 1987, pp. 110-122.

Copyright $@$ by SIAM. Unauthorized reproduction of this article is prohibited. 